Portland State University

PDXScholar

1983

\title{
Pragmatic conversational skills of young adults in normal, EMR, and TMR classrooms
}

Jane Nicholson

Portland State University

Follow this and additional works at: https://pdxscholar.library.pdx.edu/open_access_etds

Part of the Interpersonal and Small Group Communication Commons, Mental and Social Health Commons, and the Speech and Hearing Science Commons Let us know how access to this document benefits you.

\section{Recommended Citation}

Nicholson, Jane, "Pragmatic conversational skills of young adults in normal, EMR, and TMR classrooms" (1983). Dissertations and Theses. Paper 3279.

https://doi.org/10.15760/etd.3270

This Thesis is brought to you for free and open access. It has been accepted for inclusion in Dissertations and Theses by an authorized administrator of PDXScholar. Please contact us if we can make this document more accessible: pdxscholar@pdx.edu. 
AN ABSTRACT OF THE THESIS OF Jane Nicholson for the Master of Science in Speech Communication, with an emphasis in SpeechLanguage Pathology, presented June 27, 1983.

Title: Pragmatic Conversational skills of Young Adults in Normal, EMR, and TMR Classrooms.

APPROVED BY THE MEMBERS OF THE THESIS COMMITTEE:

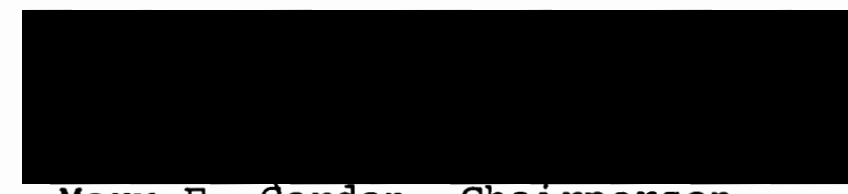

Mary E. Gordon, Chairperson

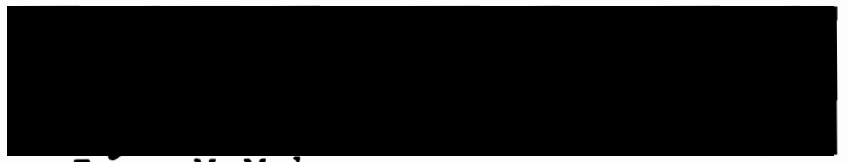

Joan McMahon

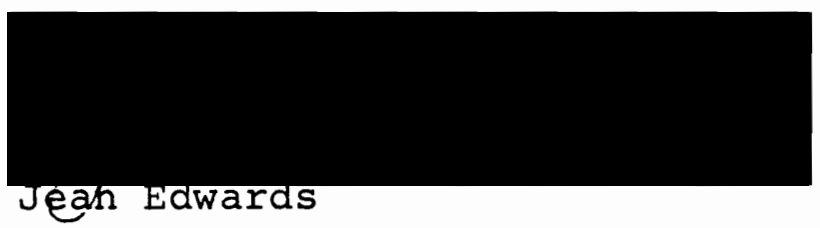

Current language theory focuses on how a person communicates within a context (Bates, 1976a). A person's communicative competence depends on how effectively she translates her cognitive and social knowledge into linguistic forms to interact in the specific situation, following pragmatic rules (Prutting, 1982b). Thus, in order to assess a person's language ability accurately, the clinician needs to assess 
pragmatic skills as well as cognitive, social, and linguistic skills. A person's pragmatic development culminates in the ability to participate in a conversation (Rees, 1978).

Speech-language pathologists work with a variety of populations, including the developmentally delayed. Research has revealed that persons with retardation acquire language in a normal, but delayed pattern. In addition, studies have shown that the language delay is related to degree of cognitive delay or retardation. Research on the pragmatic development in persons with retardation indicates that these people demonstrate some pragmatic skills, although general trends of abilities and deficits have not been determined as yet. Specifically, this author did not find any clear data comparing the pragmatic conversational behaviors of subjects with different degrees of retardation with the conversational behaviors of normal subjects. This research could provide more information about a developmental pattern for conversational behaviors as well as additional data on the relationship between developmental delay and language development.

The questions posed in this study were: 1) How do the overall performances of young adults in normal, educable mentally retarded (EMR), and trainable mentally retarded (TMR) classrooms compare when evaluated by the Pragmatic Protocol during a conversational interaction? and 2) How do the performances of young adults in normal, EMR, and TMR classrooms compare with respect to utterance act, propositional act, and illocutionary/perlocutionary act when evaluated by the 
Pragmatic Protocol during a conversational interaction?

Thirty-three young adults, aged 13 to 21 years, participated in the study. Ten were enrolled in a normal high school program, eleven in an EMR, and twelve in a TMR high school program. The investigator observed and assessed each subject using the Pragmatic Protocol in a conversation with an interactor. The interactor followed a loose script designed to elicit pragmatic strategies. The investigator recorded the subject's behaviors as appropriate or inappropriate for each of the 32 behaviors on the Protocol.

Results were analyzed by comparing the number of appropriate behaviors in each sample for total score, utterance act score, propositional act score, and illocutionary/perlocutionary act score. The data revealed significant differences on overall performance across the normal, EMR, and TMR samples. For the utterance act scores, there were significant differences between the normal and EMR groups and between the normal and TMR groups, but not between the EMR and TMR groups. For the propositional act scores, there were significant differences between the normal and TMR samples and between the EMR and TMR samples. There was a significant difference between the normal and EMR sample when using a lenient analysis (LSD) but not when using a rigorous analysis (Scheffé). In the illocutionary/perlocutionary act, there were significant differences across all three samples. In summary, the results showed that normal, EMR, and TMR young adults generally vary predictably in their overall 
conversational skills with normal students using mostly appropriate behaviors, EMR students using fewer and TMR students using the fewest number of appropriate behaviors. 


\author{
PRAGMATIC CONVERSATIONAL SKILLS OF \\ YOUNG ADULTS IN \\ NORMAL, EMR, AND TMR CLASSROOMS
}

by

JANE NICHOLSON

A thesis submitted in partial fulfillment of the requirements for the degree of

\author{
MASTER OF SCIENCE IN SPEECH COMMUNICATION: \\ with and emphasis in \\ SPEECH-LANGUAGE PATHOLOGY
}

\author{
Portland State University \\ 1983
}


TO THE OFFICE OF GRADUATE STUDIES AND RESEARCH:

The members of the Committee approve the thesis of Jane Nicholson presented June 27, 1983.

Mary E.JGordon, Chairperson

Joan Mclyanon

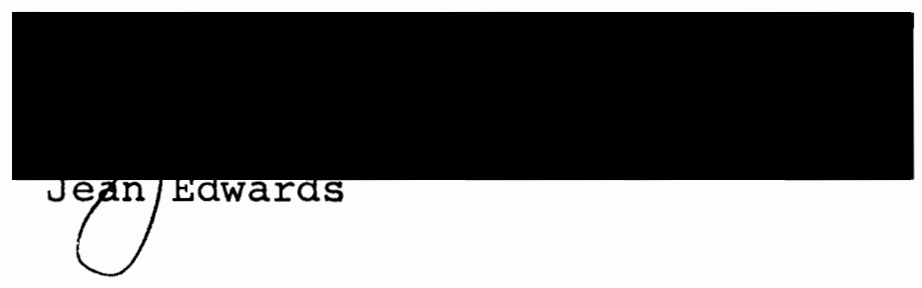

APPROVED :

'Theodore G. Grove, Chair, Department of Speech Communication

Stanley E. Rauch, Dean, Graduate Studies and Research 


\section{ACKNOWLEDGEMENTS}

This project is the result of the support and input from many people. Sincere appreciation to the following people for their professional help:

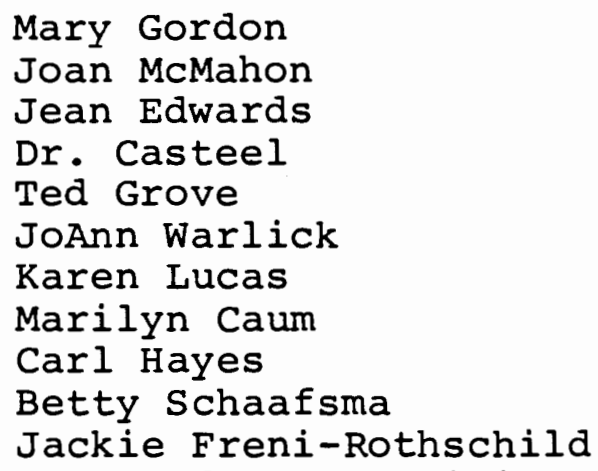

Special thanks and love to JoAnn, Kathy, Debbie, and Karen for the bonds we share. Much love and gratitude to my family, Joe Yoder, Everett and Dorothy Nicholson, and Eldon and Mary Yoder for their support and belief in education. 
TABLE OF CONTENTS

Page

ACKNOWLEDGEMENTS

LIST OF TABLES

vi

LIST OF FIGURES

vii

CHAPTER

I INTRODUCTION AND STATEMENT OF PURPOSE • • . 1

Statement of Purpose . . . . . . . . 3

Definition of Terms . . . . . . . . 3

II REVIEW OF THE LITERATURE . . . . . . . 6

Introduction $\cdot \cdot \cdot \cdot \cdot \cdot \cdot \cdot \cdot \cdot \cdot \cdot \cdot \cdot \cdot \cdot \cdot 6$

Current Framework of the Communication
Process

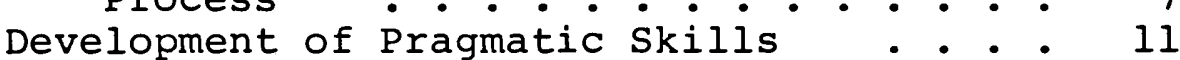

Prerequisite Behaviors for Commun-

ication ........... . 12

Development ......... . 12

Assessment ........... 13

Pragmatic Functions of Language . . 13

Development .......... 14

Assessment . . . . . . . . . . . 15

Conversational Rules and Stylistic

Variations . . . . . . . . 16

Development .......... 17

Assessment . . . . . . . . . . 18

Conversational Abilities of Young

Adults with Retardation ... . 21

Structured Communication Tasks . . 23

Observational Approach ...... . 26

Summary and Implications . . . . . 30

III METHODS . . . . . . . . . . . 32

Subjects .............. 32

Selection Criteria ........ 32

Evaluation Instrument ........ . 33

Procedures .............. . 34

Establishment of Reliability . . . 34 
Page

Investigation Procedures . . - . 35

Data Scoring and Analysis . . . . 38

IV RESULTS AND DISCUSSION

Results •. . . . . . . . . . . 39

Utterance Act .. . . . . . . 43

Propositional Act . . . . . . . 48

Illocutionary/Perlocutionary Act • . 51

Discussion . . . . . . . . . . . 57

Utterance Act . . . . . . . . 59

Propositional Act . . . . . . . 60

Illocutionary/Perlocutionary Act . . 61

Informal observations . . . . . 63

V SUMMARY AND IMPLICATIONS . • . • • • • • . 66

Summary •. . . . . . . . . . . 66

Clinical Implications . . . . . . . 68

Research Implications . . . . . . 69

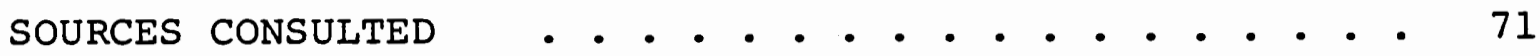

APPENDICES • • • • • • • • • • • • • • • • • 76 


\section{LIST OF TABLES}

TABLE

Page

I Inter-Judge Reliability Information . • • • 36

II Intra-Judge Reliability Information . . . . . 36

III Total Scores: Ranges, Means, and Standard Deviations ............. . . 40

IV Trend Analysis of Variance for Total Scores . 42

V Group Contrasts of Total Scores . . . . . . . 42

VI Utterance Act Scores: Ranges, Means, and Standard Deviations . . . . . . . . .

VII Trend Analysis of Variance for Utterance Act Scores . . . . . . . . . . . .

VIII Group Contrasts of Utterance Act Scores . • .

IX Propositional Act Scores: Ranges, Means, and Standard Deviations

$X$ Trend Analysis of Variance for Propositional Act Scores ............... 50

XI Group Contrasts of Propositional Act Scores .

XII Illocutionary/Perlocutionary Act scores: Ranges, Means, and Standard Deviation . .

XIII Trend Analysis of Variance for Illocutionary/ Perlocutionary Act Scores . . . . . .

XIV Group Contrasts of Illocutionary/Perlocutionary Act Scores 


\section{LIST OF FIGURES}

FIGURE

Page

1. Graph of the mean total scores of the three intelligence groups . . . . . . . .

2. Graph of the mean utterance act scores of the three intelligence groups _. . . . 44

3. Percent of each sample using appropriate strategies for utterance act behaviors .

4. Graph of the mean propositional act scores of the three intelligence groups . . . . . 49

5. Percent of each sample using appropriate strategies for propositional act behaviors

6. Graph of the mean illocutionary/perlocutionary act scores of the three intelligence groups.

7. Percent of each sample using appropriate strategies for illocutionary/perlocutionary act behaviors 


\section{CHAPTER I}

\section{INTRODUCTION AND STATEMENT OF PURPOSE}

A shift in the philosophical bases of speech-language pathology has recently occurred (Prutting, 1982a). Historically, language theory initially emphasized syntax, followed by semantics; currently, pragmatics is receiving much attention (Bates, 1976b). Bloom and Lahey (1978) respectively define these three aspects as the form, content, and use of language.

With the advent of pragmatics, emphasis is now placed on an individual as a communicator. More and more, speechlanguage pathologists look at a person's communication skills as they are used in interactions in various environments (Bates, 1976a; Holland, 1980). The level of communication competence is determined by how effectively an individual synthesizes "social and cognitive knowledge, linguistic rules, and pragmatic rules" to interact with other people (Prutting, 1982b). Thus, in planning language intervention, pragmatic development must be considered if communication skills are to be improved.

The study of pragmatic development has been organized into three major skill areas: 1) prerequisites for language; 2) language functions; and 3) conversational rules and stylistic variations. Speech-language pathologists assess these 
pragmatic areas in a variety of ways, including checklists of prerequisite behaviors (Bates, 1976a; McLean and SnyderMcLean, 1978), language sample analyses and tests to examine language functions (Dore, 1974; Halliday, 1977; Holland, 1980; Wiig, 1983), and language sample analyses for evaluating conversational skills (Miller, 1978; Prutting, 1982c). Currently, there is a need to investigate pragmatic development within normal and language-disordered populations in order to identify pragmatic deficits and plan realistic intervention goals. Speech-language pathologists intervene with many different language-disordered populations, including the developmentally delayed.

Due to recent deinstitutionalization and mainstreaming, persons with mental retardation have more opportunities to interact in the community (desilva and Faflak, 1976). How well and to what extent they interact depends upon their communicative competence. This means they need to develop their pragmatic language skills to achieve their needs, express their thoughts, and establish social relationships.

Research indicates that individuals with developmental delay use language to make their daily needs and thoughts known (Bedrosian and Prutting, 1978; Owings and McManus, 1980). Minimal research, however, has been done to look at their social conversation skills within a broad pragmatic framework. Furthermore, this author found no studies which compared pragmatic conversational abilities across the different degrees of mental retardation (mild, moderate, severe). 
This lack of data may be due, in part, to the fact that a systematic means of describing pragmatic behaviors required for conversation has not existed until recently. With the use of a conversational analysis such as the pragmatic protocol (Prutting, 1982c), it is now possible to investigate and compare the pragmatic conversational abilities of persons with normal intelligence, mild-moderate retardation, and moderate-severe retardation.

\section{Statement of Purpose}

The purpose of this study was to utilize the Pragmatic Protocol to investigate the conversational abilities in three samples of young adults/adolescents, i.e., normal functioning, mild-moderate delay, moderate-severe delay. The questions this investigation sought to answer were:

1. How do the overall performances of young adults in normal, educable mentally retarded (EMR), and trainable mentally retarded (TMR) classrooms compare when evaluated by the Pragmatic Protocol during a conversational interaction?

2. How do the performances of young adults in normal, EMR, and TMR classrooms compare with respect to the utterance act, propositional act, and illocutionary/perlocutionary act when evaluated by the Pragmatic Protocol during a conversational interaction?

\section{Definition of Terms}

The following are operational definitions of specific terms used in this investigation:

Communicative competence: the ability to adapt effectively to different contexts and to exchange messages with a 
listener (Ervin-Tripp, 1977; Hymes, 1971). According to Holland (1980), communicative competence involves the "understanding of how language functions, its social conventions, who is worth listening to, when to speak, and when to listen."

Context: minimally includes a "language user's beliefs and assumptions about temporal, spatial, and social settings; prior, ongoing, and future actions (verbal and nonverbal), and the state of knowledge and attentiveness of those participating in the social interaction at hand" (Ochs, 1979).

Conversation/social interaction: an event in which two or more people interact with each other, verbally and nonverbally, following an apparent system for both parties to focus on a similar topic, exchange turns, and perform speech acts (Myers, 1979).

Illocutionary act: the speaker's intentions (Hassan, 1982).

I.Q.: Intelligence Quotient.

Mental retardation: as defined by the American Association on Mental Deficiency, "significantly subaverage intellectual functioning existing concurrently with deficits in adaptive behavior, and manifested during the developmental period" (Grossman, 1973) . The term, developmental delay, will be used as a synonym for mental retardation in this investigation.

For the purposes of this study, educable mentally retarded (EMR) refers to students enrolled in an EMR program and whose IQ scores are in the mild-moderate range of 50-80. Trainable mentally retarded (TMR) refers to students enrolled in a multi-handicapped program and whose IQ scores are below 50 in the moderate-severe range.

Pragmatics: "the rules governing the use of language in context" (Bates, 1967a).

Perlocutionary act: the effect the speaker's intentions have on the listener (Hassan, 1982).

Propositional act: involves the linguistic component of a message consisting of a word, phrase, or sentence. Eisenson (1975) credits Jackson for defining a proposition as "a meaningful arrangement of speaker-formulated words, or a meaningful unit of speech." 
Speech act: as initially described by Austin (1962) and Searle (1969), the process through which a speaker goes to convey a message to a listener. This includes the expressed and implied intentions exchanged between speaker and listener.

Utterance act: the act of saying words (Searle, 1969). 
CHAPTER II

REVIEW OF THE LITERATURE

\section{Introduction}

In the middle 1970's, a focus on pragmatics was initiated by the profession of speech-language pathology. With current pragmatic theory, the emphasis in the literature has changed from studying an individual's language development as isolated sentences or phrases to analyzing how a child uses language to communicate and affect others' behavior (Bates, 1976b; Seibert and Oller, 1981).

Pragmatic theory incorporates phonologic, syntactic, and semantic comporents, but goes one step further, focusing on the act of communicating. Pragmatics can be defined as the study of how a "child's use of a meaningful word or sentence in a particular context of space and time will determine what is achieved by saying the word or sentence" (Seibert and oller, 1981). With a pragmatic framework of language development, speech-language pathologists look at how effectively an individual uses his phonological, syntactic, and semantic skills as part of the whole communicative process. Since language acquisition is an interactive process (Dale, 1976; Halliday, 1977, 1978), a child learns how to use both language and nonverbal skills to communicate appropriately in 
a variety of different contexts.

The trend, thus, has shifted from studying language development within a child to studying how a child's language develops as he uses it in context. With the advent of pragmatics, there have been "two important shifts in theoretical perspective: one from the structure of language to its functions, the other from the child as a thinking individual to the child as a communicator" (Myers, 1979).

Before applying pragmatic theory to language disordered populations, a clinician needs to understand the communication process and normal development of pragmatic behaviors. This study will focus on the pragmatic conversational behaviors of young adults with retardation. The communication process, normal pragmatic development, and pragmatic development in persons with retardation will be discussed in this chapter.

\section{Current Framework of the Communication Process}

Harper, Wiens, and Matarazzo (1978) present a model of communicative interaction in which one person sends a message to another person through a combination of spoken language, gestures, and facial expressions. According to Austin (1962) and Searle (1969), the message serves to convey the speaker's intent to achieve an effect upon the listener. In order to respond to others' messages and formulate one's own messages, a person must integrate cognitive and social knowledge with linguistic knowledge, following pragmatic rules (Bloom and 
Lahey, 1978; Miller, 1978; Prutting, 1982b). In other words, he must know how to exchange information (cognitive and social knowledge) by using language (linguistic knowledge) considering all aspects of the specific setting (pragmatic rules).

Social and cognitive knowledge involves "the physical world and social world, including the setting, the communicative partner, and the rules for interaction" (Prutting, 1982b). Linguistic knowledge includes phonologic, syntactic, and semantic rules, while pragmatic rules involve the various dimensions of the context when applying linguistic, social, and cognitive knowledge in a specific situation. According to Dore (1979), when a child follows pragmatic rules, he reflects what his perceptions of the situation are in relation to the social system (social beliefs, values, physical surrounding, and relationship with the other person) and uses language to achieve a purpose.

Messages are conveyed through language which Bloom and Lahey (1978) define as a "code whereby ideas about the world are represented through a conventional system of arbitrary signals for communication." Halliday (1978) describes language as not consisting of isolated sentences, but rather "of text or discourse--the exchange of meanings in interpersonal contexts of one kind or another." According to Halliday, language serves two main purposes. First, "as a means of reflecting on things," language is used to share information. Secondly, "as a means of acting on things," it is used to 
establish relationships with other people, transmit feelings, and values, and influence others' behaviors. These functions were first described by Austin (1962) and Searle (1969) as Speech Act Theory.

Austin (1962) maintained that when a person says something, he is also performing an act (e.g., naming an object or event, making a commitment to do something else, relaying attitudes and feelings, telling someone else what to do, etc.). Thus, when a person speaks, he utters words in a certain order (locutionary act) to convey a certain intent or purpose (illocutionary act) which results in consequential effects upon the feelings, thoughts, and actions of the audience (perlocutionary act). Austin also stated that people follow accepted conventional procedures or rules to perform these three acts. These rules are based on social and cognitive knowledge of a specific situation. In order to know how to influence a listener's actions, a speaker needs to assess a situation cognitively and apply specific social conventions (e.g., polite forms). In summary, Austin described how a person uses his social and cognitive knowledge to interact with others.

Searle (1969) has further discussed and refined Austin's original theory to present a framework of four aspects of the speech act; i.e., the utterance act, the propositional act, the illocutionary act, and the perlocutionary act. Searle incorporated speech and language skills in the Speech Act Theory by dividing the locutionary act into the utterance act 
and the propositional act. The utterance act focuses on the speech or vocal parameters while the propositional act focuses on the syntactic structure and the semantic content of the message. A proposition (the meaning of a statement) is expressed through the utterance act (the sequence of speech sounds). With this organization, searle linked speech and language skills to the social and cognitive skills required for human interactions. The success of these interactions depends on the communicative competence of the conversational partners.

Communicative competence is the ability to adapt effectively to different contexts and to exchange messages with a listener (Ervin-Tripp, 1977; Hymes, 1971). In order to be communicatively competent, the individual needs to know "who can say what, in what way, where and when, by what means, and to whom" (Hymes, 1971).

In general, children are linguistically competent by approximately four years of age. However, they may not achieve communicative competence until young adulthood since communicative competence relies on cognitive, social, and pragmatic growth as well as language acquisition (Bloom and Lahey, 1978; Kraus and Glucksberg, 1969). As children develop, they learn how to use different forms of language depending on the situation to achieve the same goal or function (Bloom and Lahey, 1978). For example, "Would you give me a cookie?," "Give me a cookie!," and "I wish I had a cookie," may be used to achieve the same goal. A communicatively 
competent child knows which form to use in different contexts to get that cookie. In other words, he applies pragmatic rules.

In order to assess accurately a person's communication competence, speech-language pathologists need to evaluate pragmatic skills in addition to linguistic, cognitive, and social abilities. Recent research (as cited in Bloom and Lahey, 1978; Prutting, 1982c) has begun to investigate how a child develops pragmatic skills.

\section{Development of Pragmatic Skills}

Rees (1978) cautions that "there is no simple answer to the question of when the child's communicative competence begins to develop," but it appears that pragmatic skills develop concurrently with cognitive, social, and linguistic growth (Bates, 1976a, 1979b). Rees (1978) and Prutting (1982a, 1982b) both describe pragmatic development in three major periods of a child's life, i.e., the pre-linguistic stage, the early linguistic stage, and the linguistic stage. A child demonstrates communication skills even before he speaks and continues to refine them as he learns to say words and formulate sentences. His communication skills culminate in his ability to participate in a conversation. According to prutting (1982a), a child acquires the basic skills required for conversation by school-age or age five or six. Pragmatic behaviors can be organized into three main 
categories: 1) prerequisite behaviors for language skills; 2) pragmatic functions of language, also called speech acts; and 3) conversational postulates and stylistic variations. The next sections will briefly describe each of these categories, their developmental patterns, and current assessment procedures used for each category, with emphasis on conversational postulates and stylistic variations which are the focus of this study.

Prerequisite Behaviors for Communication

Bates (1979a) emphasized the links among social maturation, cognitive growth, and language acquisition. She stated that two important developments permit humans to communicate via symbols, i.e., language: 1) the "onset of communicative intentions and conventional signals" and 2) the "emergence of symbols and discovery that things have names." These components begin to emerge between the ages of nine and thirteen months. Indicators for the onset of communicative intent involve eye contact, checks for feedback, reciprocity in a common activity, and changes in signalling until the goal is met. Indicators for the emergence of symbols include the cognitive abilities of object permanence and means-end or cause-effect relationship and the linguistic ability to recognize the relationship between an object and its label or name.

Development. A child begins to develop these prerequisite behaviors before he utters his first word (Rees, 1978). 
As he learns to talk and as he moves through the Piagetiandefined stages of development (sensori-motor, pre-operational, concrete operational), he continues to acquire the cognitive and social foundations for refining his communication skills (Bates, 1976b). In addition, he realizes that his verbal and nonverbal communication affects others' behavior. He learns to do things with language.

Assessment. To this author's knowledge, there are no standardized tests for assessing these prerequisite behaviors, although Bates (1976a), McLean and Snyder-McLean (1978), and Muma (1978) present guidelines for assessing some prerequisites for language development. Bates provides an outline for evaluating the main behaviors of symbolic play, imitation, communicative intent, and tool use. McLean and Snyder-McLean provide a communication assessment profile which lists milestones in the child's cognitive, social, and linguistic development. The Muma Assessment Plan (Muma, 1978) presents a system for describing how the child perceives the world, i.e., whether the child relies on perceptual properties (typical of children below age six years) or symbolic representations (typical of children over age six to seven years) for problem solving.

\section{Pragmatic Functions of Language}

A child learns that by manipulating her spoken language and her other communicative behaviors, she can convey her intent and affect others' behavior. She can express her intent 
by using a sentence, a phrase, a single word, or a noise accompanied by a gesture. Her interactions with others become more complex as she uses language to serve more functions or speech acts. Examples of speech acts (as described in Chapey, 1981) include:

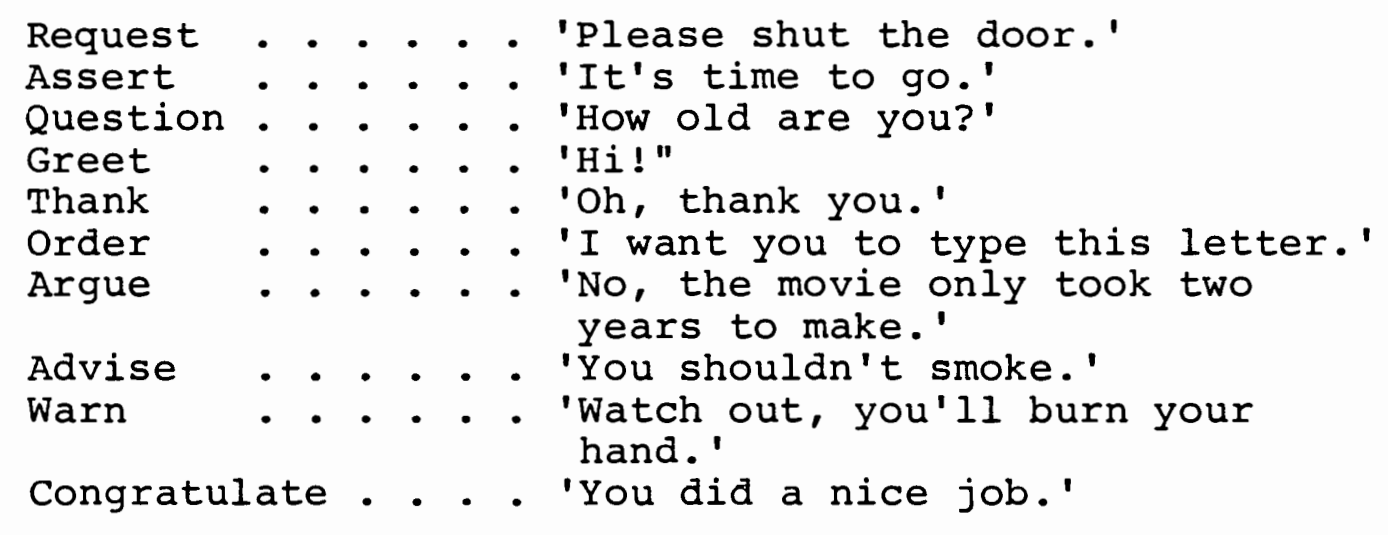

Development. Initially, a child uses language to convey a limited number of functions, e.g., request an object, request attention, get information about her world, etc. As she matures, she uses language to serve an increasing number of functions.

Bruner (1975), Dore (1975), and Halliday (1977) provide evidence that children gradually develop their facility in using a variety of speech acts. Infants mainly use imperative and declarative functions and gradually expand their repertoire to include greetings, acknowledgements, requests for answers, and protests by age three. Beyond that, "children learn to share information and provide appropriate feedback in subtle forms of communication" (Rom and Bliss, 1981).

James and Seebach (1982) substantiate this developmental pattern with their research on how children use questions. 
Between the ages of two and five years, a significant growth in the pragmatic use of questioning skills occurs. In their study, two- and three-year olds used questions mainly to seek information, while four- and five-year olds used questions to initiate or maintain a conversation, ask permission, and give a direction, as well as to seek information.

Assessment. Lists of language functions have been created by researchers such as Chapman, Jakobson, and Rees (Rees, 1978). Dore (1974, 1977, 1979) and Halliday (1977) have also provided detailed lists and definitions of language functions. In addition, they present illustrations and guidelines for eliciting and assessing the child's repertoire of speech acts. Liebman (1982) and Zimmerman (1982) thoroughly reviewed the literature and presented concise explanations of language functions. In addition, two formal tests evaluate pre-adolescents' or young adults' use of speech acts.

Let's Talk (Wiig, 1983) is an assessment tool which evaluates conversational skills within a specific situation. This inventory sets up role-play situations in which to assess an adolescent's or pre-adolescent's skills in communicating four functions: ritualizing, informing, controlling, and feeling. Each specific situation is established by giving the student verbal or pictorial cues. The student's responses are scored as appropriate or inappropriate. Total scores for each of the four speech acts can be compared with each other or with age-related criteria obtained from field test studies. In summary, this instrument provides data on a 
young adult's skills in using four speech acts in practice situations.

Holland (1980) has also recognized the need to assess the relationship between language impairment and communication competence in natural contexts. Focusing on adults with aphasia, she developed the measure of Communicative Abilities in Daily Living (CADL) to measure formally an individual's "communication skills in simulated daily life activities." The CADL had been standardized on institutionalized and noninstitutionalized aphasic and normal adults. In addition, Holland used the CADL to assess the communicative skills of adults with retardation and adults with hearing losses. The CADL focuses on assessing ten categories of communication behavior, one of which is speech acts. Gesturing, speaking, writing, reading, and understanding are evaluated in roleplay situations of daily activities such as shopping in a grocery store, making a telephone call, etc. These structured activities are presented to the subject via verbal descriptions, photographs, and "props" relevant to the context. The subject's communicative competence in each situation is scored on a three-point scale based on how effectively he gets the message across to the listener.

Conversational Rules and Stylistic Variations

As a child increases his skills in using language, he develops his ability to interact in a conversation (Bates, 1976b). Rees (1978) stated that "the conduct of conversation 
is probably the most complex as well as the most important target of the pragmatic approach to the study of language." According to Prutting (1982b), conversational competence requires that the individual be aware of the listener's perspective and the "conditions under which certain speech acts are appropriate." To manage the flow of discourse, children need to learn conversational rules such as turn allocations, topic maintenance, use of contingent queries to repair conversation breakdowns, eye gaze, physical proximity, and the protocol for interruptions. In addition, children must also learn to adapt their communication style to fit the situation and the relationship between the conversational partners. An individual learns to change his prosodic features, syntactic complexity, and semantic complexity according to his listener's skills. Some stylistic variations include polite versus informal forms, direct versus indirect requests, and adaptations in peer talk versus talk with a younger partner (Prutting, 1982b).

A child gradually learns the mechanics of conducting a conversation cooperatively with another person. He learns to follow general conversational rules of quantity, quality, relevance, and manner (Rees, 1978). In other words, he learns to communicate, in a clear manner, enough, but not too much, information which is truthful and relevant to the context.

Development. Current literature suggests the pragmatic behaviors required for conversations develop as a child matures (Miller, 1978; Rees, 1978; Seibert and Oller, 1981). 
Precursor skills, such as attracting and maintaining another's attention, directing another's attention to an object, and regulating another's behavior to achieve an end, emerge between birth and age two (Seibert and oller, 1981). Throughout his preschool years, a child becomes increasingly more adept at using different strategies to repair communication breakdowns, convey specific information, and direct and follow the topic of conversation. Prutting (1982c) cites research which indicates that basic pragmatic conversational skills are generally acquired by age six. Bates (1976a) suggests a child is able to communicate his goals within a conversational framework by age seven or eight. After this, as they develop cognitively and socially and increase their vocabulary, children refine these basic pragmatic conversational skills in more subtle ways (Bates, 1976b).

Assessment. Since conversation is a very complex process with many dimensions, it presents some problems in assessment, including: 1) on what to focus and 2) how to judge an individual's performance. These tasks become more achievable as current research reveals more information about conversational competence. In order to assess the many behaviors which occur during an interaction, it is necessary to organize the pragmatic conversational behaviors in a systematic way. Miller (1978) and Prutting (1982c) have done this in different ways.

In 1978, Miller presented a plan for analyzing chilren's pragmatic structures during meaningful communicative 
interactions with peers and adults. A child's communicative strategies are assessed during a 30-minute conversation with an adult and three children in a play situation. The assessment tool describes two main types of information: 1) the individual pattern of communication including the use of gestures, facial expression, and intonation, verbal language, syntax, and semantic categories and 2) the pattern of communication within each communicative interaction including relative dominance of speaker, turn-taking, topic maintenance/ switching, and communication breakdowns and "saves." The results of the analysis provide specific areas for intervention. The goals of intervention are to expand the number and improve the quality of a child's communicative strategies.

Miller (1978) specifies the limitations of this model. First, this analysis-intervention model is not appropriate for every child as it is best suited for preschool children at the level of symbolic play. Another limitation is that not all behaviors occur naturally during a conversational setting. In other words, while playing, a child may not demonstrate his highest level of sentence structure or his most sophisticated form of a speech act. Finally, the author points out the difficulty in accurately measuring qualitative behaviors in quantitative terms.

In contrast, the Pragmatic Protocol (Prutting, 1982c) uses a molar approach, providing a general framework for analyzing pragmatic skills during a conversation. Generally based on Austin's (1962) and Searle's (1969) Speech Act 
Theory, the Protocol is a combination of recent pragmatic taxonomies which outline verbal and nonverbal behaviors for taking both speaker and listener roles in a conversation including turntaking and initiating, maintaining, and repairing topics (Hassan, 1982). Prutting organized these behaviors into three major categories: the utterance act, the propositional act, and the illocutionary and perlocutionary acts. An interaction is observed and each of the 32 behaviors is scored as appropriate, inappropriate, or not observed. The format of the Protocol permits several means of analyzing a person's performance. First, the person's score can be recorded quantitatively as an overall score or as a score for each of the three separate categories. The person's performance may also be discussed descriptively by recording comments about the quality of his responses (e.g., how he repaired the conversation when it broke down).

As a clinical instrument, the Pragmatic Protocol was primarily designed to analyze a person's skills within a conversational dyad. As such, it provides an overview of an individual's pragmatic abilities, pinpointing specific skills to assess in depth later. The Protocol can also be used to study an individual's pragmatic communication skills across a variety of normal daily situations and contexts.

In addition, the Pragmatic Protocol can be used as a research instrument to gather data about the pragmatic conversational skills of samples of different populations. For some specific populations, becoming more socially adept or 
conversationally competent is a major goal. One such population is adolescents or young adults with mental retardation.

Conversational Abilities of Young Adults with Retardation

As discussed previously, conversation competence requires that a person integrate cognitive, social, linguistic, and pragmatic skills. If pragmatic development parallels social and cognitive development and linguistic acquisition, it would seem that persons with delayed or disordered development would demonstrate some deficits in pragmatic conversational skills as well. This study has focused on comparing the pragmatic conversational skills of young adults with delayed development with the pragmatic skills of young adults with normal development.

According to the American Association of Mental Deficiency (AAMD) definition of retardation, persons with retardation are delayed cognitively and socially (Robinson and Robinson, 1965). The AAMD's 1973 definition of retardation specifies that people with retardation demonstrate "significant subaverage intellectual functioning existing concurrently with deficits in adaptive behavior, and manifested during the developmental period" (Grossman, 1973). These cognitive and social delays affect a person's educational, vocational, and social life.

With the advent of deinstitutionalization and mainstreaming, young adults with mental retardation have more opportunities to work and socialize in their communities 
(de Silva and Faflak, 1976). One of the current goals in mainstreaming and deinstitutionalization is to maximize the opportunities "for retarded people to contact others in the surrounding community" (Berkson and Romer, 1980).

One study investigated how some of these adults function in the community. In their survey of eighteen deinstitutionalized adults with mild to borderline retardation, McDevitt, Smith, Schmidt, and Rosen (1978) found that the subjects had few social interactions and felt unsure of handling new situations, even though most of the subjects had been in the community since the late 1960's. Generally, they carried out daily living skills adequately, but they seemed to be "asocial" with social contacts centering around an activity rather than conversations with friends. These subjects expressed feelings of loneliness or isolation which were attributed to "personality deficits or environmental circumstances rather than to mental retardation or the effects of the institution."

Conversational skills are an important aspect of being accepted by and relating to other people (Camaioni, 1979) . Thus, it is important that young adults with retardation develop their social skills and communicative competence (Bates, 1980). A first step in this process is to analyze the language skills of persons with mental retardation to determine what their deficits are and then plan intervention which may help them improve their communication competence.

Reynolds and Reynolds (1979) and Robinson and Robinson 
(1965) noted that speech and language impairment is one of the most commonly occurring handicaps among mentally retarded persons. Research indicates that persons with retardation develop the form and meaning of language in a normal, but slower pattern (Bloom and Lahey, 1978; Lackner, 1976; Tamari, 1978). These results, however, "do not reflect or predict how the retarded use their language for communication" (Bedrosian and Prutting, 1978). As Halfond and Tamari (1980) noted, little research has been done on the pragmatic conversational skills of persons with retardation. Past studies have basically followed two main organizations, i.e., 1) use of structured communication tasks and 2) use of observations in natural settings (Beveridge, 1976). Studies using structured communication tasks impose certain restrictions on the communicative interaction and require specific responses from both conversational partners. As such they do not investigate general conversation skills, but do provide pieces of information about the subject's communication skills.

Structured Communication Tasks

Structured communication tasks follow two main experimental designs: a no-cost reinforcement and a barrier game design. Spradlin, Girardeau, and Corte (1967) and Hollis (1966) used a paradigm in which one subject could choose to deliver reinforcement to another subject without losing his own reinforcement. In addition, both received more rewards if the second subject communicated to the first subject what 
response to make and if the first subject responded appropriately to that communication.

Hollis' (1966) results indicated that even profoundly retarded children took the other subject's perspective and rewarded the receiver when there was no cost to themselves. As mentioned before, taking the other person's perspective is a required element of communicative competence. The findings also revealed rudimentary turntaking and topic maintenance as even severely retarded partners exchanged specific information and repaired communication breakdowns in a simplistic manner.

In Spradlin et al.'s (1967) study, twenty high level and twenty low level subjects (ages 11 to 15 years) were selected based on performance on the Parsons Language Sample and on the Wechsler Intelligence Scale for Children (WISC). The higher level receivers gave more feedback to the operators to indicate which stimuli to select, and therefore received more reinforcement than the lower level receivers. This indicates that the ability to control the environment through language correlates with intellectual level, specifically in relation to giving feedback to the other person.

The other main experimental design was a barrier game with two subjects seated on either side of a barrier. One of the subjects was shown a picture to describe clearly enough so that the listener could identify it correctly from his array of pictures. This task required the speaker to adjust his verbal description to fit the listener's cognitive and 
linguistic abilities. Longhurst (1972) found that normal adult dyads performed these tasks with few errors. Dyads of retarded adolescents performed the tasks with about 50 percent accuracy (Longhurst, 1974).

In the latter study, Longhurst (1974) also found a significant difference in both speaker and listener roles among three intelligence levels. Intelligence levels were determined by WISC or Wechsler Adult Intelligence Scale (WAIS) scores. Generally, subjects with IQ scores of 70 to 90 performed better than subjects with IQ scores of 56 to 69 , who, in turn, performed better than subjects with IQ scores of 40 to 55. Thus, again, communication skills seemed to correlate with intelligence level.

In 1975, Longhurst and Berry used this paradigm to investigate retarded adolescent speakers' responses to requests for clarification. Subjects were grouped into high, medium, and low groups. IQ's were 71 to 92,56 to 68 , and 41 to 55 , respectively. The number and type of redescriptions (new, modified, repeated, or silence) were analyzed. There were significant differences in both quantity and quality of "redescription" skills among the three intelligence levels. As before, the high group tended to perform better than the medium group which in turn performed better than the low group. While these studies present evidence that pragmatic communication skills in structured tasks are affected by level of intelligence, they do not provide specific information about conversational skills. Therefore, to assess discourse 
abilities accurately, it is necessary to observe people in actual conversations (Faine and Longtin, 1981).

Observational Approach

Studies which have investigated the spontaneous discourse abilities of mentally retarded subjects will be presented in this next section. Each of the studies analyzes specific pragmatic conversational behaviors such as those listed on the Pragmatic Protocol.

Beveridge (1976) cited an investigation by Beveridge and Berry which observed free play interactions of five special education classrooms. Results of this study indicated that the older handicapped children (ages 10 to 16 years) consistently initiated significantly more verbal interactions than younger mentally handicapped children (ages 5 to 10 years). These authors then further analyzed the older children's types of initiations according to function. They found that "there were over twice as many occurrences of giving information as asking for it, which is the reverse order found in the initiation behavior of normal children." Thus, these results suggest that as mentally handicapped children mature, they initiate discourse more frequently, although the ways they initiate conversation differ from how normal children initiate conversation.

Bedrosian and Prutting (1978) used a sociolinguistic analysis to investigate how four mentally retarded adults handled the dimensions of dominance-submission and control 
when engaged in conversations with different people. The subjects were 23 to 28 years old and attended a training center for developmentally delayed adults. Their IQ scores ranged from 29 to 36 except for one subject. This subject's IQ score was not reported, however his language and mental age scores were similar to the other subjects' scores. Each subject's conversations with his speech-language pathologist, peers, parents, and a normal six year old child were recorded and analyzed according to dominance-submission and types of control. Three of the subjects did not hold the dominant position in any of the conversations. The fourth subject, a 26 year old woman with an IQ score of 31 , held the dominant position in conversations with her peers and the child. All the subjects expressed the same types of conversational control (e.g., request for action, information, or attention) as normal adults, although these types varied as a function of the conversational partner. Bedrosian and Prutting suggested that these results present three clinical implications. First, linguistic skills or deficits do not necessarily reflect communicative competence since people with limited vocabulary and sentence length can control the topic and turntaking aspects of a conversation. Thus, it is important to assess pragmatic skills as well as syntactic, semantic, and phonologic skills. Secondly, it is also important to assess the mentally retarded person's communication skills in a variety of conversational settings. Finally, persons with mental retardation are a heterogeneous group in respect to how 
and how often they express control of a conversation.

Owings and McManus (1980) also looked at one specific pragmatic behavior, i.e., variety of speech acts or functions. They examined the types of speech acts a moderately retarded adult used in three different contexts. Results demonstrated that even a subject with a "reduced verbal productive language ability" uses a variety of functions in each context and uses the functions selectively, varying the frequency of each function to fit the context.

An educable mentally retarded child's use of pragmatic functions was explored by Halfond and Tamari (1980). These researchers used Dore's method to analyze the subjects' verbal and nonverbal intents in three different contexts. They compared the variety of speech acts used by a seven year old retarded child with those used by two normal children, one matched for chronological age and one matched for mean length of utterance. Results indicated that the mentally retarded girl's use of speech acts was more similar to the girl her own age than to the younger girl matched for utterance length. Like Owings and McManus (1980), Halfond and Tamari found that the subject with retardation varied the types of functions across different contexts.

The main limitation in the Halfond and Tamari (1980) study was the inability to code nonverbal communication completely since the transcriptions were based primarily on audiotaped recordings and since some of these behaviors did not fall into one of the specified categories. A second 
limitation which Halfond and Tamari pointed out was that this study analyzed only functions, and in order to assess discourse skills fully, the person's use of appropriate and relevant language needs to be evaluated as well. Thus, future research needs to look at the entire range of conversational skills, not just the use of pragmatic functions.

one study did look more broadly at discourse behaviors, in particular, introducing and maintaining topics. Faine and Longtin (1981) videotaped a 13 year old male with Down's Syndrome interacting with other persons: a normal male matched for chronological age, a normal male matched for mean length of utterance, (age 2 years, 10 months), and a mentally retarded male matched for mean length of utterance (age 10 years). Although context and nonverbal behaviors were recorded, only verbal utterances were analyzed. Conversational utterances were coded as either strategies used to maintain continuous discourse or to change the topic. In addition, speaker dominance was assessed by how effectively the subject used each of these strategies. Strategies for maintaining continuous discourse were incorporating the discourse topic and collaborating the discourse topic by acknowledging the previous statement or by using a topic extender. Strategies for changing the topic included introducing a new topic, re-introducing a topic, using an attention-getter prior to introducing or re-introducing a topic, and using off-target responses which are not relevant to the previous conversation and which do not introduce a topic. 
Faine and Longtin (1981) found that the 13 year old student with retardation used the same strategies as the normal children to participate in coherent continuous discourse. Additionally, he used all of the strategies except topic extenders. The authors suggested that topic extenders seem to be a more advanced conversational behavior, possibly acquired later than other strategies. The results also indicated that the subject varied his strategies for controlling the conversation depending on who his partner was.

\section{Summary and Implications}

In summary, as a child acquires and integrates linguistic skills, cognitive and social knowledge, and pragmatic rules, she becomes a more competent communicator. Her communication skills culminate in the ability to participate in a conversation with another person. It has been documented that persons with retardation demonstrate cognitive, social, and linguistic delays; however, research on mentally handicapped adults' pragmatic discourse skills has been limited. Investigations have focused on specific pragmatic behaviors rather than providing a broad overview of pragmatic abilities and deficits. Findings of past studies have indicated that people with retardation do acquire some pragmatic conversational skills, although this development appears to differ from the normal population's development. There is a need to investigate the general discourse skills of young adults with retardation. 
The Pragmatic Protocol is one means of evaluating these skills. By using the Protocol, the speech-language clinician may obtain an overview of an individual's conversational strengths and weaknesses. From this overview, the examiner can further probe specific behaviors and then plan intervention strategies aimed at improving the individual's communication skills. It is hoped that pragmatic-oriented language intervention may then improve the quality and quantity of the person's interactions with other people (Seibert and oller, 1981). 
CHAPTER III

METHODS

\section{Subjects}

Subjects were recruited from high school programs through Gresham Public Schools and Multnomah County Education Service District. Thirty-three persons who passed the screening criteria were selected to participate in the study. Twenty-three young adults/adolescents with mental retardation and ten normal young adults/adolescents, ages 13 to 21 years old, were chosen. Of the people with retardation, eleven were enrolled in an educable mentally retarded (EMR) classroom with general IQ requirements of 50-80. The other twelve were trainable mentally retarded (TMR) students with IQ scores generally 50 and below. The TMR students were enrolled in multi-handicapped $(\mathrm{M}-\mathrm{H})$ classrooms. The ten normal young adults attended regular high school classes and did not receive special education services. These students were volunteer aides in the EMR and $\mathrm{M}-\mathrm{H}$ classes.

Selection Criteria

Potential subjects with signed letters of consent were screened (Appendix A). Selection criteria included:

1. passing a pure-tone audiometric screening administered at $25 \mathrm{~dB}$ for the frequencies of 500,1000 , 
2000 , and $4000 \mathrm{~Hz}$ in one ear,

2. using oral speech,

3. using English as the primary language, and

4. having no diagnosis of any severe physical or sensory handicap such as blindness or severe Cerebral Palsy which may have penalized them disproportionately on the verbal and nonverbal parameters when compared with normal peers without severe physical handicaps.

\section{Evaluation Instrument}

The Pragmatic Protocol describes the pragmatic language skills involved in conversational interactions. Specifically, it focuses on three aspects of a communicative interaction: the utterance act, the propositional act, and the illocutionary/perlocutionary act. Each of the three categories consists of specific behaviors. In all, 32 specific behaviors are outlined in the protocol. The 13 behaviors under the utterance act include the verbal, nonverbal, vocal, and paralinguistic aspects of speech production. The 4 behaviors under the propositional act involve the linguistic aspects of communication. The 15 behaviors listed under the illocutionary/perlocutionary act focus on the actual interchange and how smoothly the participants interact. Appendix B lists the taxonomy, modality, description and coding, and references for each of the behaviors.

The Pragmatic Protocol (Appendix C) provides a means of 
qualitatively assessing a subject's interactions as "appropriate," "inappropriate," or "not observed." These judgments are made in reference to the communicative setting and the relationship between the conversational partners.

\section{Procedures}

Establishment of Reliability

Prior to conducting the study, inter- and intra-judge reliability of the investigator's use of the Pragmatic Protocol was achieved at a level of .85 or better. To determine reliability, the investigator and two judges observed $10 \mathrm{mi}-$ nute videotapes of conversational dyads and compared protocol results. The judges involved in the reliability training included a clinical supervisor with a Master of Science degree and a Certificate of Clinical Competency awarded by the American Speech-Language-Hearing Association, the investigator, and another graduate student in the Speech and Hearing Sciences Program at Portland State University. Results between the investigator and the other graduate student and between the investigator and the clinical supervisor were compared and reliability was calculated using the following formula: agreements

$\overline{\text { disagreements + agreements }}$

Before the training sessions, each judge read the Prutting (1982b) journal article on pragmatics, the pragmatic Protocol (Appendix B), and a handout describing the behaviors on the protocol (Appendix C). At the training sessions, the 
judges used the protocol to evaluate two trial videotapes. Appendices $D$ and $E$ describe the procedure used to evaluate a subject's behaviors according to the parameters on the Protocol. Results were compared and discussed. The three judges then used the Protocol to evaluate the subjects on four videotapes. Discrepancies in scoring were discussed and the rating procedure was repeated with other dyads. This procedure was continued until inter-judge reliability for each dyad was .85 or better.

The mean reliability rating between the investigator and the clinical supervisor was .93. The mean reliability rating between the investigator and the other graduate student was .96. Table I displays complete reliability data. Intra-judge reliability rating of .85 or better was similarly achieved by comparing the investigator's scoring on a set of four videotapes at the training session with the investigator's scoring on those same tapes approximately ten weeks later, just prior to conducting the study. Intra-judge reliability data are presented in Table II.

\section{Investigation Procedures}

The investigator screened potential subjects by: 1) administering a hearing screening to determine the student's hearing acuity and 2) asking the teacher and student questions, and observing student in classroom setting to determine the student's physical handicaps, primary language, and use of oral speech. Thirty-three students who met all 
TABLE I

INTER-JUDGE RELIABILITY INFORMATION

\begin{tabular}{lll}
\hline ubject & Investigator & Investigator \\
& and Judge \#1 & and Judge \#2 \\
\hline
\end{tabular}

\begin{tabular}{lcc}
$\begin{array}{lc}\text { Child with Language } \\
\text { Disorders }\end{array}$ & .94 \\
Normal Adult & .94 & .97 \\
Adult with Retardation & .88 & .94 \\
Normal Child & .97 & 1.00 \\
\hline & Mean & .93 \\
\hline
\end{tabular}

TABLE II

INTRA-JUDGE RELIABILITY INFORMATION

\begin{tabular}{lr} 
Child with Language Disorders & .94 \\
Normal Adult & 1.00 \\
Adult with Retardation & .94 \\
Normal Child & 1.00 \\
\hline & Mean \\
\hline
\end{tabular}

screening criteria were selected to participate in the study. After an interim of approximately two weeks, the investigator returned to the school to collect the data with an 
interactor, a graduate student in speech-language pathology. Each of the thirty-three subjects interacted on a one-to-one basis with the interactor in an informal setting. A school staff member introduced the subject to the interactor and the investigator and asked the subject to talk with them for awhile.

The subject, interactor, and investigator went to an empty room in the building (see Appendix $F$ for floor plans and seating arrangement). Distractions were kept to a minimum by not allowing other persons in the area during the interaction. The subject and the interactor sat facing each other at an angle slightly greater than $90^{\circ}$ and approximately 4-6 feet apart. The investigator stated that she had some paperwork to do, excusing herself from the conversation, and sat approximately 10 feet from the dyad. The investigator "busied herself" with some papers, while inconspicuously observing the interaction.

The interactor followed a "loose script" for each conversation. The script was structured so as to permit the subject to demonstrate his/her skills for specific behaviors on the Pragmatic Protocol. In order to maintain a somewhat natural conversation, the script specified only strategies used by the interactor, not a complete dialogue (see Appendix G) After making a judgment on each of the 32 parameters, the investigator briefly joined the conversation and then dismissed the subject. Each interaction lasted 10 to $15 \mathrm{mi}-$ nutes. When the subject left the room, the investigator 
recorded the subject's conversational behaviors on the Pragmatic Protocol form.

\section{Data Scoring and Analysis}

Each subject was evaluated using the Pragmatic Protocol. Data were analyzed using parametric statistics. A straight single factor analysis of variance (Anova), a test of LeastSignificant Difference (LSD), and a Scheffé post hoc were used to determine whether there were significant differences in total scale scores among the three samples. A trend analysis was done by graphing the mean total scale scores for each of the three samples. The performances of the three samples were further compared by completing these statistical analyses on utterance act scores, propositional act scores, and illocutionary/perlocutionary act scores. 
CHAPTER IV

RESULTS AND DISCUSSION

$\underline{\text { Results }}$

The purpose of this study was to utilize the pragmatic Protocol to investigate the conversational skills of three samples of young adults: those in a regular high school program, those in an EMR classroom, and TMR students in a multihandicapped (M-H) program. Each student was observed in a conversation with an interactor. The student's conversational skills were coded as appropriate or inappropriate for each of 32 behaviors listed on the Pragmatic Protocol. All raw scores appear in Appenidx $\mathrm{H}$.

The first question posed was: How do the overall performances of young adults in normal, EMR, and TMR classrooms compare when evaluated by the Pragmatic Protocol during a conversational interaction? Each group's raw score ranges, means, and standard deviations were computed and are presented in Table III. With 32 points possible, the ten normal subjects' scores ranged from 31 to 32 with a mean of 31.90 and a standard deviation (S.D.) of 0.32 . The scores of the eleven EMR subjects ranged from 18 to 32 with a mean of 24.45 and a S.D. of 4.68. The twelve TMR students scored from 8 to 27 with a mean of 18.00 and a S.D. of 5.91. To demonstrate 
TABLE III

TOTAL SCORES: RANGES, MEANS, AND STANDARD DEVIATIONS

\begin{tabular}{lcccc}
\hline Sample & $\mathrm{N}$ & $\begin{array}{l}\text { Range } \\
\text { possible })\end{array}$ & Mean & Standard Deviation \\
\hline Normal & 10 & $31-32$ & 31.90 & 0.32 \\
EMR & 11 & $18-32$ & 24.45 & 4.68 \\
TMR & 12 & $8-27$ & 18.00 & 5.91 \\
\hline
\end{tabular}

graphically how the overall performances varied, the means have been plotted in Figure 1 .

The total score distributions were tabulated and analyzed using a one-way fixed trend analysis of variance (Anova). Table IV displays the results. This analysis revealed the difference among the groups to be statistically significant with an $F$ value of $26.19(p<.001)$. The test for trend analysis also revealed a significant linear trend with an $F$ value of $52.30(p<.001)$.

Group comparisons were performed using three orthogonal group contrasts (Table V). The contrast between the normal and EMR students produced a significant $\underline{t}$-value of 3.799 $(p=.001)$. The contrast between the normal and TMR students produced a significant $\underline{t}$-value of $7.237(p<.001)$. Finally, the contrast between the EMR and TMR students produced a significant t-value of $3.447(p<.01)$. Since there were unequal 


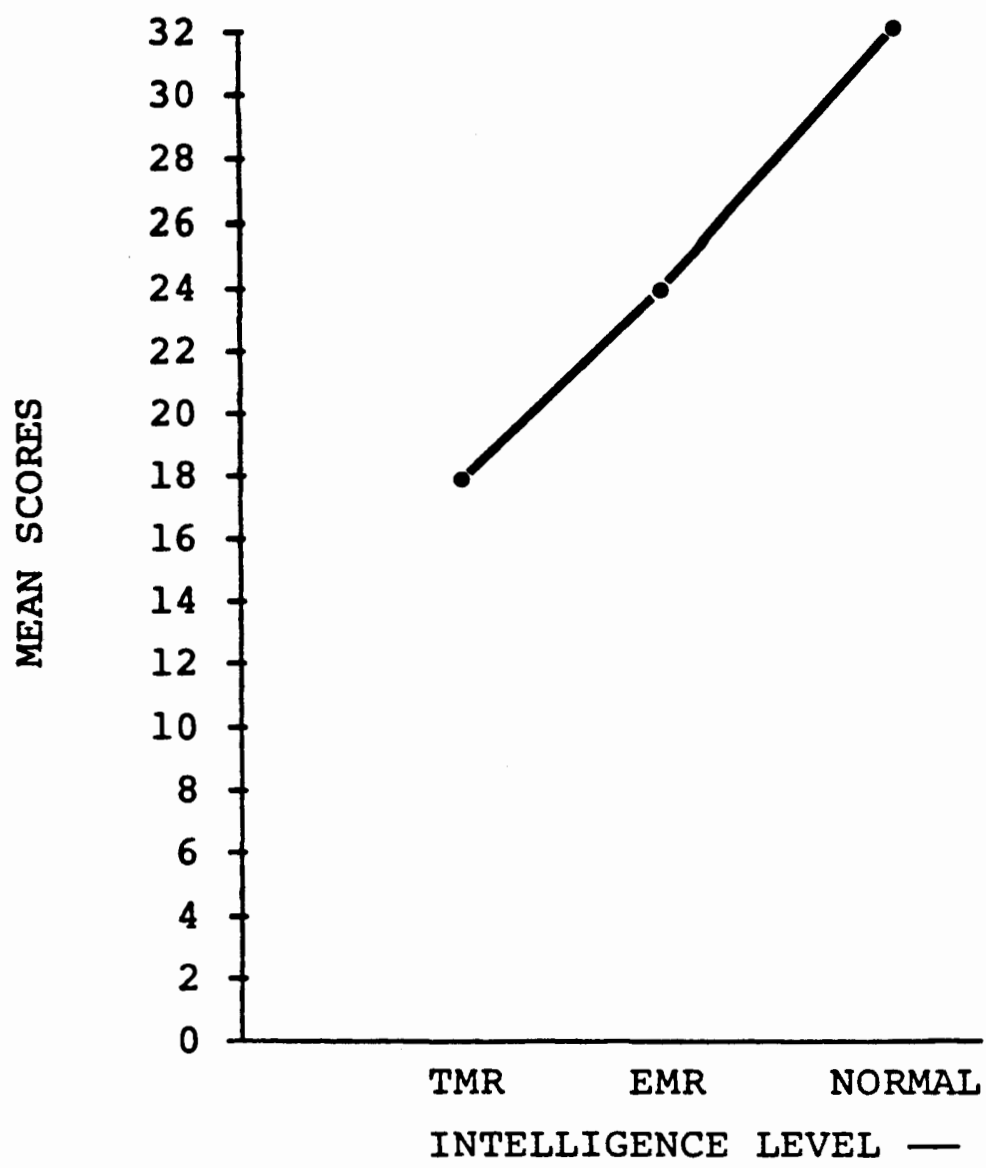

Figure 1. Graph of the mean total scores of the three intelligence groups.

numbers in the cells and there was a significant difference in overall performance, aposteriori tests of Least-Significant Difference (LSD), a lenient statistical test, and the Scheffé post hoc test, a rigorous statistical test, were also done to further evaluate whether specific pairs of group performances were significantly different. Again, the differences between the groups were significant at or beyond the .01 level, with the difference between the normal and TMR samples at the .001 significance level for both the Scheffé and the LSD tests. Thus, significant differences were found 
TABLE IV

TREND ANALYSIS OF VARIANCE FOR TOTAL SCORES

\begin{tabular}{|c|c|c|c|c|c|}
\hline Source of Variation & $\underline{\text { SS }}$ & $\underline{d f}$ & $\underline{\text { MS }}$ & $\underline{\mathbf{F}}$ & $\underline{P}$ \\
\hline Total & 1657.64 & $\underline{32}$ & & & \\
\hline Between Groups & 1054.01 & 2 & 527.00 & 26.19 & $<.001 *$ \\
\hline $\begin{array}{l}\text { Linear Term } \\
\text { (Weighted) }\end{array}$ & 1052.21 & 1 & 1052.21 & 52.30 & $<.001 *$ \\
\hline $\begin{array}{l}\text { Deviation from } \\
\text { Linear }\end{array}$ & 1.80 & 1 & 1.80 & 0.09 & 0.767 \\
\hline Within Groups & 603.63 & 30 & 20.12 & & \\
\hline
\end{tabular}

* Significant

TABLE V

GROUP CONTRASTS OF TOTAL SCORES

\begin{tabular}{|c|c|c|c|c|c|}
\hline Groups & $\underline{t}$ & $\frac{\text { Anova }}{\text { df }}$ & $\underline{P}$ & $\frac{L S D}{\underline{P}}$ & $\frac{\text { Scheffé }}{\underline{P}}$ \\
\hline $\begin{array}{c}\text { Normal } \\
\text { vs. } \\
\text { EMR }\end{array}$ & 3.799 & 30 & $.001 *$ & $.001 *$ & $.01 *$ \\
\hline $\begin{array}{c}\text { Normal } \\
\text { vs. } \\
\text { TMR }\end{array}$ & 7.237 & 30 & $<.001 *$ & $.001 *$ & $.001 *$ \\
\hline $\begin{array}{c}\text { EMR } \\
\text { VS. } \\
\text { TMR }\end{array}$ & 3.447 & 30 & $.002 *$ & $.01 *$ & $.01 *$ \\
\hline
\end{tabular}

* Significant 
in the overall performances of the three groups.

A second question was posed to compare further how the samples performed on the three main sections of the protocol. The second question was: How do the performances of young adults in normal, EMR, and TMR classrooms compare with respect to the utterance act, propositional act, and illocuionary/perlocutionary act when evaluated by the Pragmatic Protocol during a conversational interaction? Thus, three indices of conversational competence (utterance act skills, propositional act skills, and illocutionary/perlocutionary act skills) were examined across the three intelligence levels. For each of the indices, raw score ranges, means, and S.D.S were computed. The score distributions were tabulated and analyzed using an Anova. The difference among the groups' performances were further analyzed with the lenient LSD and the rigorous scheffé. In addition, each of the behaviors within each act was tabulated and presented descriptively as what percentage of each sample used appropriate strategies to demonstrate strengths and weaknesses.

\section{Utterance Act}

of the 13 possible points, the normal subjects each scored 13, with a mean of 13 and S.D. Of 0.00 . The EMR subjects' scores ranged from 8 to 13 , with a mean of 10.64 and S.D. of 2.11. The TMR subjects' scores ranged from 7 to 13 , with a mean of 10.33 and S.D. of 1.72. These data are presented in Table VI with the mean scores plotted in Figure 2. 
TABLE VI

UTTERANCE ACT SCORES: RANGES, MEANS, AND STANDARD DEVIATIONS

\begin{tabular}{lcccc}
\hline Sample & N & $\begin{array}{l}\text { Range } \\
\text { possible })\end{array}$ & Mean & Standard Deviation \\
\hline Normal & 10 & 13 & 13.00 & 0.00 \\
EMR & 11 & $8-13$ & 10.64 & 2.11 \\
TMR & 12 & $7-13$ & 10.33 & 1.72 \\
\hline
\end{tabular}

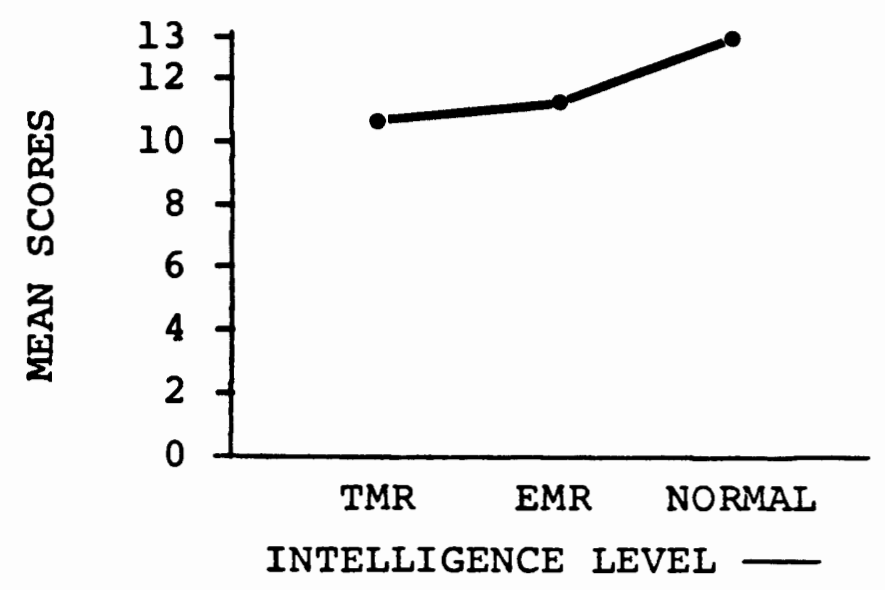

Figure 2. Graph of the mean utterance act scores of the three intelligence groups.

An analysis of variance showed the between-groups difference was significant with an $F$ value of $8.71(p=.001)$. In addition, the test for trend analysis revealed a significant linear trend with an $F$ value of 14.41 ( $p<.001)$. Table VII displays this information.

Group comparisons were also done (Table VIII). Using Anova, the contrast between the normal and EMR students 
TABLE VII

\section{TREND ANALYSIS OF VARIANCE \\ FOR UTTERANCE ACT SCORES}

\begin{tabular}{|c|c|c|c|c|c|}
\hline Source of Variation & $\underline{\text { SS }}$ & $\underline{d f}$ & $\underline{\mathrm{MS}}$ & $\underline{F}$ & $\underline{P}$ \\
\hline Total & 122.06 & 32 & & & \\
\hline Between Groups & 44.85 & 2 & 22.42 & 8.71 & $.001 *$ \\
\hline $\begin{array}{l}\text { Linear Term } \\
\text { (Weighted) }\end{array}$ & 37.09 & 1 & 37.09 & 14.41 & $<.001 *$ \\
\hline $\begin{array}{l}\text { Deviation from } \\
\text { Linear }\end{array}$ & 7.76 & 1 & 7.76 & 3.02 & .09 \\
\hline Within Groups & 77.21 & 30 & & & \\
\hline
\end{tabular}

Significant

\section{TABLE VIII}

GROUP CONTRASTS OF UTTERANCE ACT SCORES

\begin{tabular}{|c|c|c|c|c|c|}
\hline Groups & $\underline{t}$ & $\frac{\text { Anova }}{\mathrm{df}}$ & $\underline{P}$ & $\frac{\mathrm{LSD}}{\underline{\mathrm{P}}}$ & $\frac{\text { Scheffe }}{P}$ \\
\hline
\end{tabular}

Normal

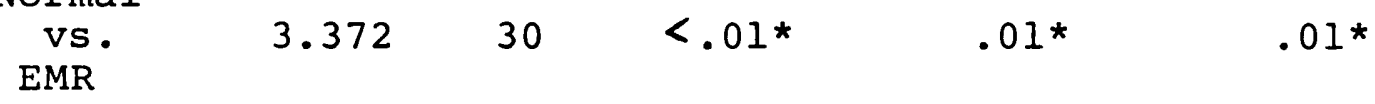

Normal

\begin{tabular}{|c|c|c|c|c|c|}
\hline $\begin{array}{l}\text { VS. } \\
\text { TMR }\end{array}$ & 3.882 & 30 & $.001 *$ & $.001 *$ & $.01 *$ \\
\hline EMR & & & & & \\
\hline $\begin{array}{l}\text { VS. } \\
\text { TMR }\end{array}$ & .453 & 30 & .654 (NS) & NS & NS \\
\hline
\end{tabular}

Significant NS Not Significant 
resulted in a significant t-value of $3.372(p<.01)$. The Scheffé and LSD tests also revealed a significant difference at the .01 level. Anova showed a significant difference between the normal and TMR samples with a t-value of 3.882 $(p=.001)$; significant differences were also shown by the Scheffé $(p=.01)$ and the LSD $(p=.001)$. When comparing the EMR and TMR subjects, a $\underline{t}$-value of 0.453 was obtained. The difference between the EMR and TMR samples was not significant using any of the three statistical tests. In summary, for the utterance act, the differences between the normal and EMR samples and between the normal and TMR samples were significant while the difference between the EMR and TMR sample was not.

Each group's performance on the utterance act was further analyzed descriptively by determining the percentage of specific behaviors used appropriately by each group, as an indication of the pragmatic strengths and weaknesses within each intelligence group (Figure 3). All normal subjects were appropriate for each of the 13 behaviors. All EMR and TMR subjects were appropriate on fluency, physical proximity, and gestures. Less than 80 percent of the EMR subjects were appropriate on speech intelligibility, vocal intensity, vocal quality, hand/arm movements, and eye gaze. Less than 80 percent of the TMR students were appropriate on speech intelligibility, vocal quality, prosody, and eye gaze. Upon visual inspection, the three groups seemed to differ most notably on speech intelligibility with 100 percent of the normal group, 
= Normal

$\square=E=E M R$

II11111=TMR
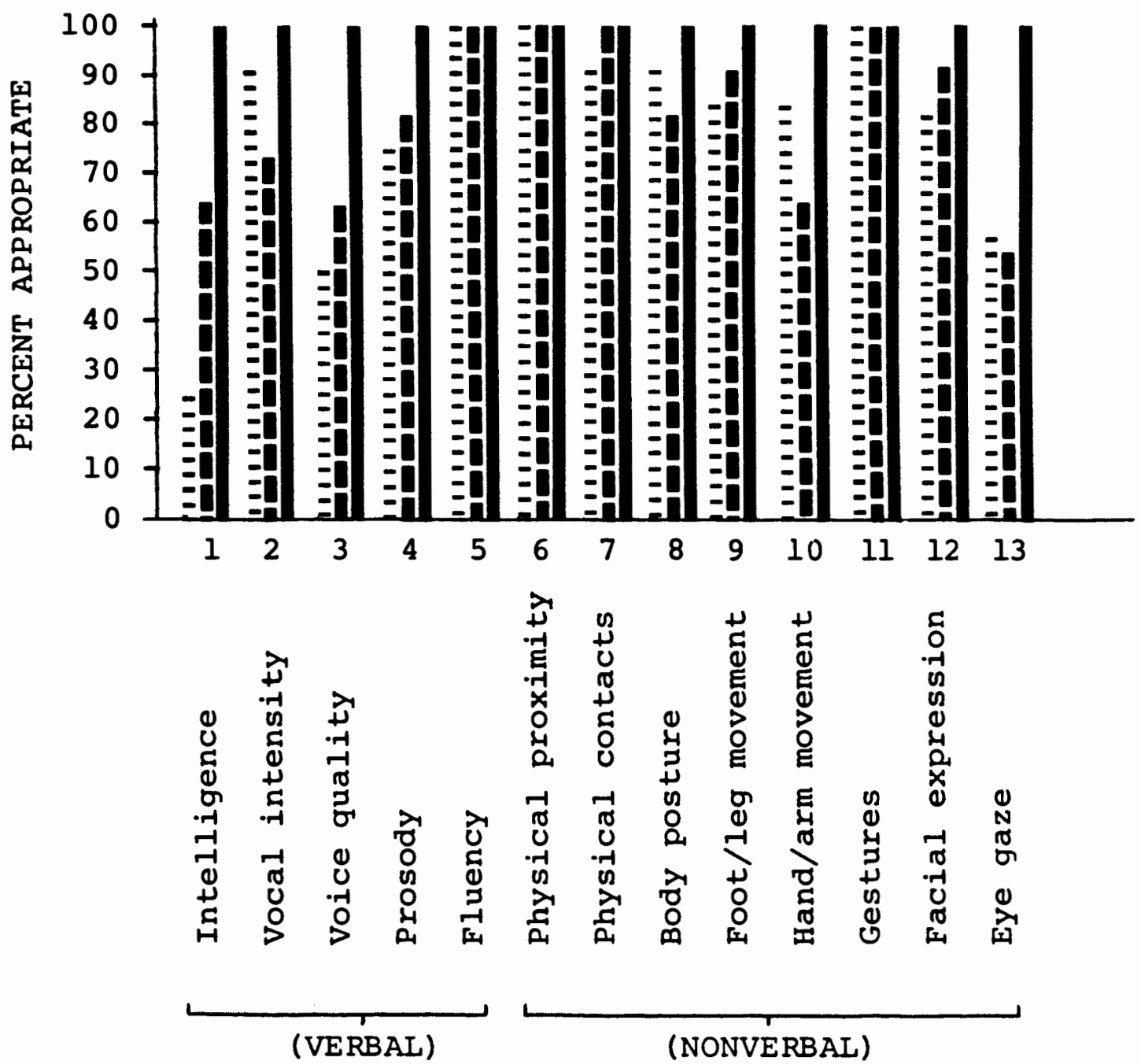

ERBAL)

(NONVERBAL)

Figure 3. Percent of each sample using appropriate strategies for utterance act behaviors. 
64 percent of the EMR sample, and 25 percent of the TMR sample using intelligible speech appropriately. Results also indicated that approximately 50 percent of both EMR and TMR samples demonstrated inappropriate eye gaze. In general, they used too little eye contact.

Propositional Act

of 4 points possible on the propositional act category, the normal subjects all scored 4 with a mean of 4.00 and S.D. of 0.00. The EMR subjects scored from 1 to 4 with a mean of 3.00 and S.D. of 1.10, while the TMR group scored from 0 to 4 with a mean of 1.25 and S.D. of 1.14. Table IX presents these data. The mean scores are plotted on Figure 4 to demonstrate the linear progression across intelligence groups.

An analysis oi variance revealed a significant betweengroups difference with an F value of 24.62 ( $p<.001)$. A significant linear trend of $F=48.06$ ( $p<.001$ ) was found by the test for trend analysis (Table $\mathrm{X}$ ).

Group comparisons were done by using Anova, Scheffé, and LSD tests (Table XI). On the Anova, the contrast between the normal and EMR samples was significant beyond the .05 level with a $\underline{t}$-value of 2.447 . The LSD test also revealed a significant difference at the .05 level; however, the Scheffé indicated that the difference between the normal and EMR samples was not significant. The difference between the normal and the TMR groups was at or beyond the .001 significance 
TABLE IX

PROPOSITIONAL ACT SCORES: RANGES, MEANS, AND STANDARD DEVIATIONS

\begin{tabular}{lcccc}
\hline Sample & N & $\begin{array}{l}\text { Range } \\
\text { possible })\end{array}$ & Mean & Standard Deviation \\
\hline Normal & 10 & 4 & 4.00 & 0.00 \\
EMR & 11 & $1-4$ & 3.00 & 1.10 \\
TMR & 12 & $0-4$ & 1.25 & 1.14 \\
\hline
\end{tabular}

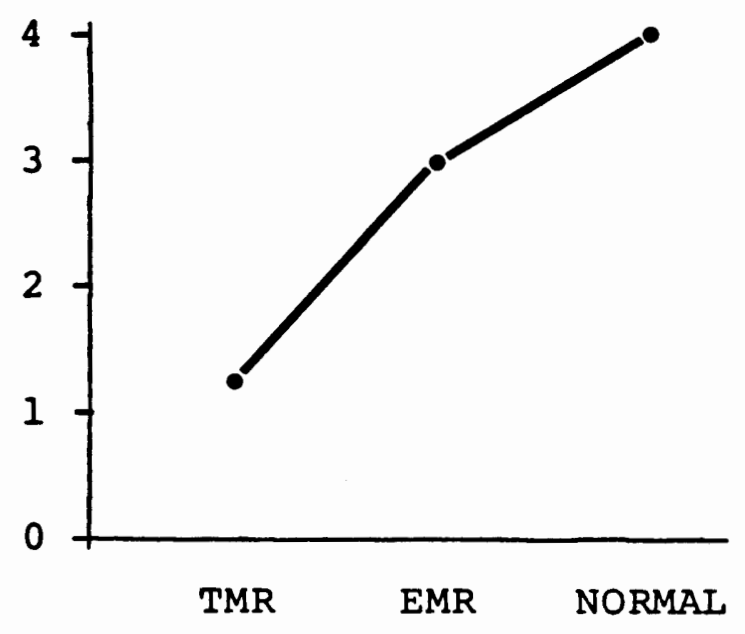

INTELLIGENCE LEVEL

Figure 4. Graph of the mean propositional act scores of the three intelligence groups.

level for all three tests with an Anova $\underline{t}$-value of 6.866 . The difference between the EMR and TMR subjects was also at or beyond the .001 significance level for all three tests with an Anova t-value of 4.482. In short, for the propositional act, the differences between the normal and EMR, between the normal and TMR, and between the EMR and TMR samples 
TABLE X

TREND ANALYSIS OF VARIANCE FOR PROPOSITIONAL ACT SCORES

\begin{tabular}{|c|c|c|c|c|c|}
\hline Source of Variation & $\underline{\text { SS }}$ & $\underline{d f}$ & $\underline{\text { MS }}$ & $\underline{F}$ & $\underline{\mathrm{P}}$ \\
\hline Total & $\underline{69.33}$ & 32 & & & \\
\hline Between Groups & 43.08 & 2 & 21.54 & 24.62 & $<.001 *$ \\
\hline $\begin{array}{l}\text { Linear Term } \\
\text { (Weighted) }\end{array}$ & 42.05 & 1 & 42.05 & 48.06 & $<.001 *$ \\
\hline $\begin{array}{l}\text { Deviation from } \\
\text { Linear }\end{array}$ & 1.03 & 1 & 1.03 & 1.18 & .29 \\
\hline Within Groups & 26.25 & 30 & .88 & & \\
\hline
\end{tabular}

* Significant

TABLE XI

GROUP CONTRASTS OF PROPOSITIONAL ACT SCORES

\begin{tabular}{lccccc}
\hline Groups & $\underline{t}$ & $\frac{\text { Anova }}{\mathrm{df}}$ & $\underline{\mathrm{P}}$ & $\frac{\mathrm{LSD}}{\underline{\mathrm{P}}}$ & $\frac{\text { Scheffé }}{\underline{\mathrm{P}}}$ \\
\hline $\begin{array}{c}\text { Normal } \\
\text { VS. } \\
\text { EMR }\end{array}$ & 2.447 & 30 & $<.05^{*}$ & $.05^{*}$ & NS \\
$\begin{array}{c}\text { Normal } \\
\text { VS. } \\
\text { TMR }\end{array}$ & 6.866 & 30 & $.000^{*}$ & $.001^{*}$ & $.001^{*}$ \\
$\begin{array}{c}\text { EMR } \\
\text { VS. } \\
\text { TMR }\end{array}$ & 4.482 & 30 & $.000^{*}$ & & $.001^{*}$ \\
\hline
\end{tabular}

* Significant

NS Not Significant 
were all significant except when using the scheffé to contrast the normal and EMR groups.

The results were further analyzed by tabulating the percentage of each group which used the specific behaviors appropriately (Figure 5). All normal subjects used the four propositional act behaviors appropriately. Only one behavior, varying of communicative style, was used appropriately by at least 80 percent of the EMR sample. None of the behaviors were used appropriately by at least 80 percent of the TMR sample. Upon visual inspection, there were striking differences between the EMR and TMR performances: 1) 73 percent of EMR students, but only 25 percent of TMR students, used specific and accurate words; 2) 73 percent of EMR subjects, but only 25 percent of TMR subjects, used appropriate word order; 3) 64 percent of the EMR sample, but only 17 percent of the TMR sample, handled new and given information appropriately; and 4) 91 percent of EMR subjects, while only 58 percent of TMR subjects, varied their communicative style appropriately.

\section{Illocutionary/Perlocutionary Act}

of 15 possible points, the normal group's scores ranged from 14 to 15 with a mean of 14.90 and S.D. of 0.32 . The EMR scores ranged from 7 to 15 with a mean of 10.82 and S.D. of 2.79. The scores of the TMR sample ranged from 1 to 14 with a mean of 6.42 and S.D. of 4.14 . This information is summarized in Table XII with the mean scores plotted on Figure 6 . An analysis of variance showed the between-groups difference 
$=$ Normal

man =EMR

$1111111=T M R$

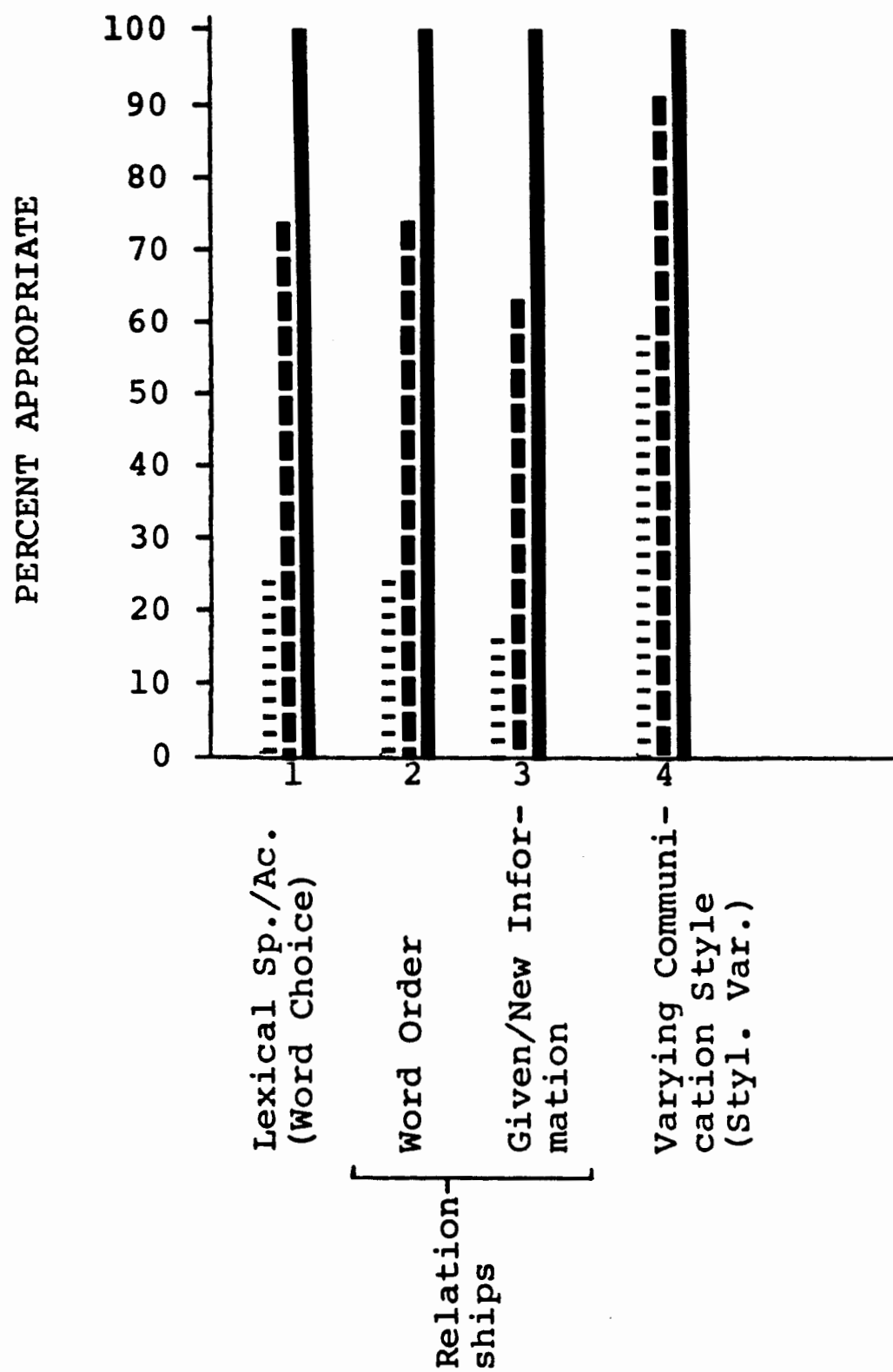

Figure 5. Percent of each sample using appropriate strategies for propositional act behaviors.

was significant with an $F$ value of $22.14(p<.001)$. The test for trend analysis revealed a significant linear trend with an $F$ value of $44.26(p<.001)$. Table XIII shows these data. 
TABLE XII

ILLOCUTIONARY/PERLOCUTIONARY ACT SCORES: RANGES, MEANS, AND STANDARD DEVIATIONS

\begin{tabular}{lcccc}
\hline Sample & N & $\begin{array}{l}\text { Range } \\
\text { possible })\end{array}$ & Mean & Standard Deviation \\
\hline Normal & 10 & $14-15$ & 14.90 & .32 \\
EMR & 11 & $7-15$ & 10.82 & 2.79 \\
TMR & 12 & $1-14$ & 6.42 & 4.14 \\
\hline
\end{tabular}

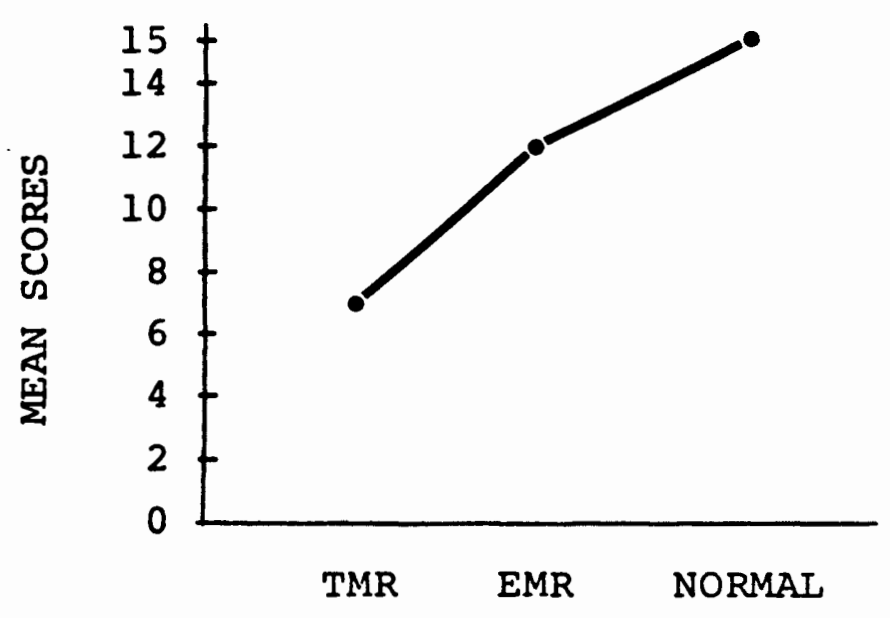

INTELLIGENCE LEVEL

Figure 6. Graph of the mean illocutionary/perlocutionary act scores of the three intelligence groups.

Group comparisons were also run (Table XIV). Using an Anova, the contrast between the normal and EMR students resulted in a significant $\underline{t}$-value of $3.129(p=.004)$. Using the scheffé test, the significance level was .05 and with the LSD test, the significance level was .01. The difference between the normal and TMR subjects was significant at or beyond 
TABLE XIII

TREND ANALYSIS OF VARIANCE FOR ILLOCUTIONARY/

PERLOCUTIONARY ACT SCORES

\begin{tabular}{|c|c|c|c|c|c|}
\hline Source of Variation & $\underline{\text { sS }}$ & $\underline{d f}$ & $\underline{\mathrm{MS}}$ & $\underline{F}$ & $\underline{\mathrm{P}}$ \\
\hline Total & 662.18 & $\underline{32}$ & & & \\
\hline Between Groups & 394.73 & 2 & 197.36 & 22.14 & $<.001 *$ \\
\hline $\begin{array}{l}\text { Linear Term } \\
\text { (Weighted) }\end{array}$ & 394.54 & 1 & 394.54 & 44.26 & $<.001 *$ \\
\hline $\begin{array}{l}\text { Deviation from } \\
\text { Linear }\end{array}$ & .19 & 1 & .19 & .02 & .886 \\
\hline Within Groups & 267.45 & 30 & 8.92 & & \\
\hline
\end{tabular}

* Significant

TABLE XIV

GROUP CONTRASTS OF ILLOCUTIONARY/

PERLOCUTIONARY ACT SCORES

\begin{tabular}{|c|c|}
\hline Groups & $t \quad \frac{\text { Anova }}{d f}$ \\
\hline
\end{tabular}

Normal

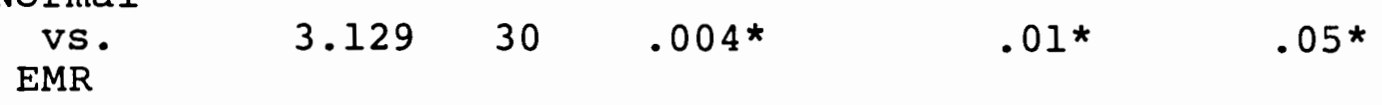

Normal

$\begin{array}{cccccc}\text { VS. } & 6.636 & 30 & .000 * & .001 * & .001 \text { * } \\ \text { TMR } \\ \begin{array}{c}\text { EMR } \\ \text { VS. } \\ \text { TMR }\end{array} & 3.532 & 30 & .001 * & .01 * & .01 * \\ \end{array}$


the .001 level for all three statistical tests, with an Anova t-value of 6.636. Comparison of the EMR and TMR performances revealed a significant difference also with an Anova $\underline{t}$-value of $3.532(p=.001)$ and significance levels of .01 for both the Scheffé and LSD analyses. In summary, for the illocutionary/perlocutionary act, the differences between the normal and EMR groups, between the normal and TMR groups, and between the EMR and TMR groups were all significant.

A descriptive analysis of the percent of subjects using appropriate behaviors for each of the behaviors is presented in Figure 7. Ninety percent or more of the normal subjects used each of the behaviors appropriately. Eighty percent or more of the EMR students used appropriate behaviors for speech act pair analysis, topic selection, response, repair/revision, interruption/overlap, feedback to speaker, and contingency. The EMR subjects were more consistently inappropriate on variety of speech acts (45 percent appropriate), topic introduction (55 percent appropriate), topic change (45 percent appropriate), and quantity/conciseness (45 percent appropriate). Less than 80 percent of the TMR students used appropriate strategies for each of the behaviors. The higher percentages of appropriateness included variety of speech acts (67 percent), response ( 75 percent), interruption/overlap 175 percent), and feedback to speaker (67 percent). The lower percentages were speech act pair analysis ( 17 percent), pause time ( 8 percent), and quantity/conciseness ( 8 percent). 
$=$ Normal

m =EMR

11111 =TMR
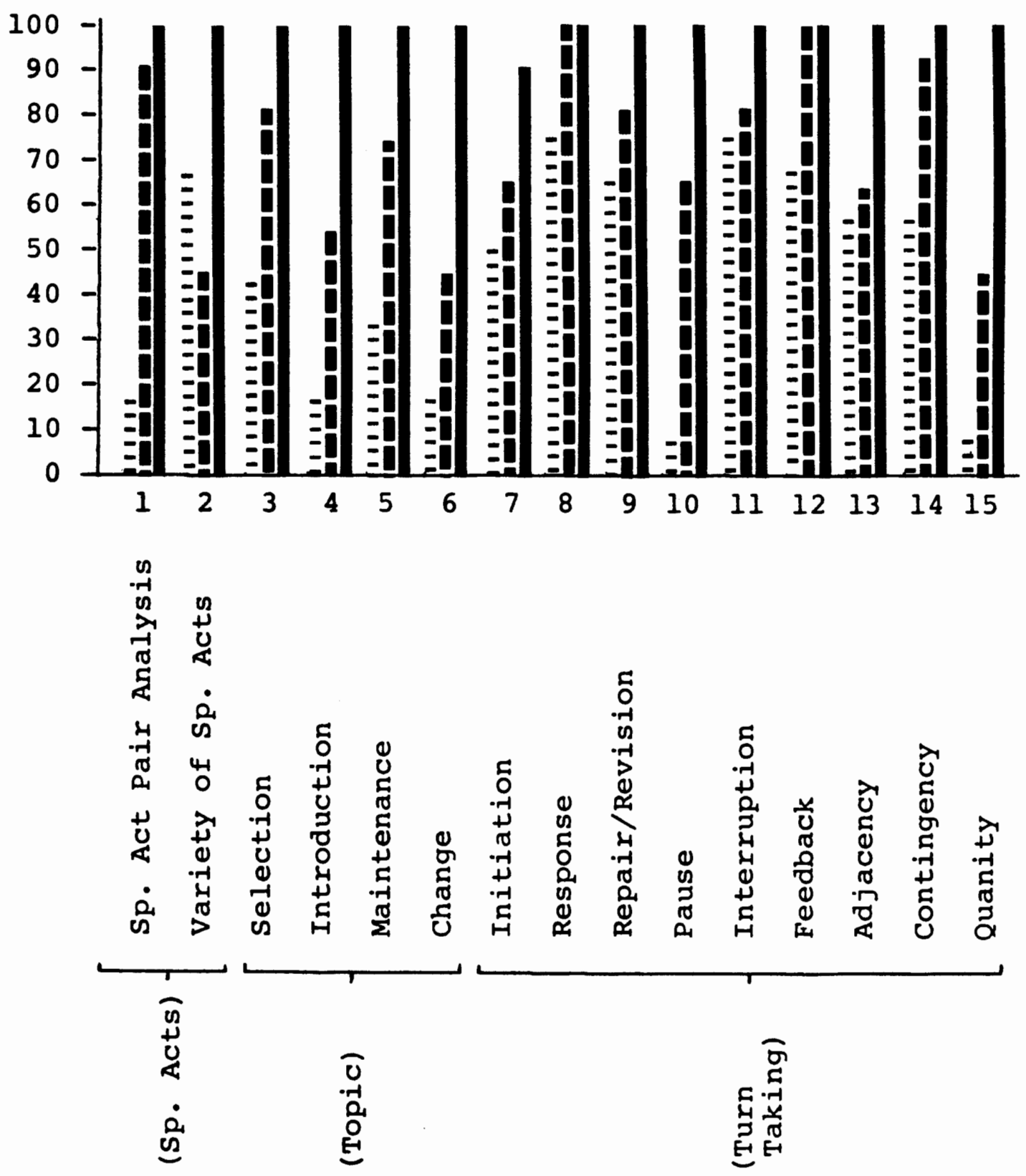

Figure 7. Percent of each sample using appropriate strategies for illocutionary/perlocutionary act behaviors. 


\section{$\underline{\text { Discussion }}$}

The findings in this study indicate that overall pragmatic conversational skills did vary among young adults in TMR, EMR, and normal high school programs. The normal high school students generally used appropriate behaviors, indicating that conversational competency is acquired by adolescence. The EMR and TMR results suggested that pragmatic conversational skills generally decrease with retardation level, agreeing with previous studies conducted by Longhurst (1972; 1974), Longhurst and Berry (1975), and Spradlin, Girardeau, and Corte (1967).

Although there were significant differences between groups, there were no definite cut-off scores for each group. The ranges of scores for the three different samples overlapped. Similar to subjects in previous studies, all the retarded adolescents in this study displayed some appropriate communication skills. There was, however, a range of abilities within each group. In fact, there were overlapping scores across the three groups with one EMR student using more appropriate behaviors than one of the normal students and five of the TMR students scoring higher than the lowest EMR score. This overlap may be explained by a number of factors such as subject selection, familiarity with the investigator, and individual differences among the subjects.

This study used classroom placement as the only criterion for determining intelligence level. To be eligible for 
the EMR classroom, the students generally have IQ scores in the range of 50 to 80 . Students in the TMR program generally have IQ scores below 50. Other factors such as social adaptability, academic skills, and behavior frequently influence the placement decision. As a result, some of the higher level TMR students may function like EMR students and some of the lower ability EMR students may function like students in the TMR program. This indicates there may be an overlap of skills in EMR and TMR adolescents. The students' histories may have differed widely in their past conversational experiences and education. In addition, elimination of nonverbal students may have caused the TMR sample to be higher functioning than the typical TMR population.

The samples' performances were further analyzed by examining how they performed on the three sections of the Pragmatic Protocol. This research found that persons with retardation do use some appropriate conversational strategies such as variety of speech acts, topic introduction and maintenance, turn-taking, and varying of communicative style. This finding concurs with studies conducted by Bedrosian and Prutting (1978), Faine and Longtin (1981), Halfond and Tamari (1980), Hollis (1966), and Owings and McManus (1980). As with the total scores, there were overlapping scores across adjacent intelligence samples. However, in general, all the normal students used mostly appropriate strategies for the situation, while the EMR students used fewer and the TMR students used the fewest appropriate strategies. There were seven 
exceptions to this in the utterance act and one exception in the illocutionary/perlocutionary act.

Utterance Act

In the utterance act, the results suggested that both the EMR and TMR young adults performed poorer than the normal adolescents. There was, however, no significant difference between the EMR and TMR groups. Even though more of the EMR subjects performed better than or as well as the TMR subjects on ten of the thirteen parameters, more of the TMR subjects performed better than the EMR students on vocal intensity, body posture, and hand/arm movements. This lack of significant difference between EMR and TMR may be due to the design of the study, the abilities and past experiences of these particular students, or the nature of the utterance act behaviors.

Since nonverbal and multi-handicapped students were eliminated from the study, more TMR than EMR students were disqualified as subjects. Therefore, the TMR sample did not represent their population as accurately as the EMR sample represented theirs. In fact, the TMR subjects used in this study were all verbal and seemed to have higher level conversation skills than their nonverbal classmates. Thus, the TMR subjects reflected the abilities at the higher end of the TMR range whereas the EMR subjects were typical of the entire range of EMR abilities in their classrooms.

Another possible explanation is that the EMR students 
were less familiar with the investigator. Even though they did not interact with the investigator during the conversation, they possibly were more conscious of her presence, and therefore demonstrated their shyness or nervousness by using a quiet voice, leaning forward and rocking, and using excessive and inappropriate arm movements. For instance, one EMR student covered his face with his arms. Since most of the TMR students had known the investigator previously, fewer appeared to be "nervous" about the situation, even though the investigator was 10 feet away and not directly interacting with the subject. These individual differences must be considered. As Bedrosian and Prutting (1978) pointed out, persons with retardation are a heterogeneous population and caution should be used when making generalizations regarding their communication skills.

A third explanation may be that utterance act tasks (speech and nonverbal communication) are not as abstract and therefore are acquired more easily than propositional act and illocutionary/perlocutionary act behaviors. For example, even a pre-linguistic child learns appropriate prosody and uses it in jargon. Therefore, these EMR students and high functioning TMR students may have acquired these skills similarly on a basic level, but have not refined them like the normal students have.

Propositional Act

On the linguistic component of the Protocol, the 
propositional act, the normal students performed better than the EMR students who, in turn, performed better than the TMR students. This is consistent with Longhurst's $(1972 ; 1974)$, Longhurst and Berry's (1975), and Spradlin et al.'s (1967) hypothesis that general communication skills correlate with level of intelligence. In addition, the results of this study support Lackner's (1976) and Tamari's (1978) earlier findings which indicate that persons with retardation develop syntactic and semantic skills in a normal, but delayed pattern.

\section{Illocutionary/Perlocutionary Act}

On fourteen of the fifteen illocutionary/perlocutionary parameters, the EMR subjects tended to be more appropriate than the TMR subjects. Most of the EMR students took both speaker and listener roles, while most of the TMR students tended to be more passive, responding to the interactor's questions, but not taking an active part in controlling the conversation. These findings are similar to Bedrosian and Prutting's (1978) results in which three of the four subjects did not hold the dominant position in any conversational setting. Possible explanations for the TMR subjects' passivity included: 1) they have not acquired the cognitive prerequisites; 2) they have not had sufficient conversational experiences; 3 ) they did not have the propositional (linguistic) skills such as vocabulary, syntax, and referencing; or 4) they have been under strict stimulus control, being conditioned to 
not saying anything unless spoken to.

This passivity also influenced how the TMR adolescents were judged on topic selection, introduction, maintenance, and change, as well as turn-taking behaviors. Since they tended to participate only by responding minimally to the interactor's questions, their scores on topic maintenance and turn-taking were also lower than the EMR sample's scores. As in Faine and Longtin's (1981) study, it appears that persons with retardation demonstrate difficulty with topic maintenance and the use of topic extenders.

It must be noted, however, that not all TMR students were passive and too quiet. Some were judged inappropriate due to excessive talking. These students were inappropriate by using too little pause time, introducing irrelevant topics, and not maintaining one topic adequately. In addition, TMR students tended to divulge more personal information (e.g., their financial status, family drinking habits) to a stranger than did the EMR and normal students. It should also be noted that some of the TMR students did participate in the discourse with appropriate topic and turn-taking behaviors.

The normal and EMR adolescents handled communication breakdowns more appropriately than the TMR students by requesting clarification from the interactor and by revising their own statements if necessary. On the other hand, only 50 percent of the TMR subjects requested clarification when the interactor deliberately "mumbled." The other half continued to talk, ignoring the interactor's comment or 
pretending to understand and going on with what they were saying. In turn, the interactor tried to further elicit a request for clarification by saying something with her mouth closed. Even with this obvious communication breakdown, some TMR students did not request the interactor to clarify what she had said. There were also quality differences in how the TMR students revised or repaired their statements. While the normal and EMR subjects tended to change the wording in their statements, the TMR subjects tended to either repeat their sentence, decrease their speaking rate, or ignore the interactor's request for revision.

In summary, results suggested that illocutionary/perlocutionary act skills corresponded with intelligence level. The main exception to this was in the variety of speech acts. Sixty-seven percent of TMR students, but only 45 percent of the EMR students, used an appropriate range of functions during the conversation. This may have been due to shyness on the part of the EMR students, the TMR students' higher need to request help in finding words and following directions, or some additional reason not recognized at this time.

\section{Informal Observations}

In addition to observations based on data analysis, the investigator and interactor made informal observations about the nature of the interaction and the instrument. One informal observation was that both the interactor and the investigator seemed to adjust their vocabulary and syntax according 
to the individual's apparent communicative ability. For example, since the TMR students did not respond as well to general comments and open-ended questions, the interactor used more direct questions with them than with the normal and EMR students. The investigator also changed her directions, especially in closing the conversation and dismissing the students. Since the TMR students tended to ask fewer, if any, questions about the study, and did not realize when the conversation was finished, the experimenter used shorter sentences and more gestures with them than with EMR and normal subjects. Thus, these findings supported Shatz and Gelman's (1973) contention that people adjust to their listeners. This interactor and investigator adjustment may have influenced some of the results and the reader is cautioned about these differences.

Another informal observation involved the nature of the Pragmatic Protocol. The Protocol uses a molar approach for analyzing conversational skills. It seems to function best as a screening device. As such, it provides an overview of a person's strengths and weaknesses. It is not a discreet assessment of the quality of those behaviors. Since there are no specific criteria for each of the behaviors (e.g., no time limit for appropriate eye contact), the judgment of appropriateness is subjective based on how smoothly the interaction went. In the future, investigators may choose to modify the Protocol to meet their needs better. Some recommendations for modifying the Protocol include: 1) use a scale rather 
than a dichotomy of appropriate/inappropriate (e.g., the CADL and the Porch Index of Communicative Ability respectively use a 3- and 15-point scale for recording responses); 2) condense the protocol form to fit onto one or one and a half pages with a close-knit lined grid which would be easier to read and allow a long space for comments in place of the "no opportunity to observe" column; 3) include an "emerging" or "inconsistent" column; 4) record the number of appropriate and number of inappropriate responses for each behavior rather than making one overall judgment of appropriateness. 


\section{CHAPTER V}

\section{SUMMARY AND IMPLICATIONS}

\section{Summary}

Current language theory focuses on how a person communicates within a context (Bates, 1976a). A person's communicative competence depends on how effectively she translates her cognitive and social knowledge into linguistic forms to interact in the specific situation, following pragmatic rules (Prutting, 1982b). Thus, in order to assess a person's lan-. guage ability accurately, the clinician needs to assess pragmatic skills as well as cognitive, social, and linguistic skills. A person's pragmatic development culminates in the ability to participate in a conversation (Rees, 1978).

Speech-language pathologists work with a variety of populations, including the developmentally delayed. Research has revealed that persons with retardation acquire language in a normal, but delayed pattern. In addition, studies have shown that the language delay is related to degree of cognitive delay or retardation. Research on the pragmatic development in persons with retardation indicates that these people demonstrate some pragmatic skills, although general trends of abilities and deficits have not been determined as yet. Specifically, this author did not find any clear data comparing 
the pragmatic conversational behaviors of subjects with different degrees of retardation with the conversational behaviors of normal subjects. This research could provide more information about a developmental pattern for conversational behaviors as well as additional data on the relationship between language development and developmental delay . The questions posed in this study were: 1) How do the overall performances of young adults in normal, educable mentally retarded (EMR), and trainable mentally retarded (TMR) classrooms compare when evaluated by the Pragmatic Protocol during a conversational interaction? and 2)How do the performances of young adults in normal, EMR, and TMR classrooms compare with respect to utterance act, propositional act, and illocutionary/perlocutionary act when evaluated by the pragmatic Protocol during a conversational interaction?

Thirty-three young adults, aged 13 to 21 years, participated in the study. Ten were enrolled in a normal high school program, eleven in an EMR, and twelve in a TMR high school program. The investigator observed and assessed each subject using the Pragmatic Protocol in a conversation with an interactor. The interactor followed a loose script designed to elicit pragmatic strategies. The investigator recorded the subject's behaviors as appropriate or inappropriate for each of the 32 behaviors on the Protocol.

Results were analyzed by comparing the number of appro$4 \mathrm{C}$

priate behaviors in each sample for total score, utterance act score, propositional act score, and illocutionary/ 
perlocutionary act score. The data revealed significant differences on overall performance across the normal, EMR, and TMR samples. For the utterance act scores, there were significant differences between the normal and EMR groups and between the normal and TMR groups, but not between the EMR and TMR groups. For the propositional act scores, there were significant differences between the normal and TMR samples and between the EMR and TMR sample. There was a significant difference between the normal and the EMR sample when using a lenient analysis (LSD) but not when using a rigorous analysis (Scheffé). In the illocutionary/perlocutionary act, there were significant differences across all three samples. In summary, the results showed that normal, EMR, and TMR young adults vary predictably in their overall conversational skills with normal students using mostly appropriate behaviors, EMR students using fewer and TMR students using the fewest number of appropriate behaviors.

\section{Clinical Implications}

These results provide preliminary data supporting the theory that pragmatic development parallels cognitive, social, and linguistic development. By adolescence, normal students acquire appropriate strategies for participating in conversations. Students with retardation demonstrate some appropriate and some inappropriate conversational strategies. The number and type of inappropriate behaviors seem to be related to the degree of retardation. The trends of strengths and 
weaknesses within the normal, EMR, and TMR samples provide general guidelines of what to expect within each of the three intelligence ranges. The results of this study, particularly the percentage tables, may be used as preliminary normative data with which to compare a client's behaviors. The clinician can then use this information to make realistic prognoses and to plan pragmatic-oriented intervention aimed at improving the client's communicative competence. In addition, scripts such as the one in Appendix G may be used as assessment and intervention aids to elicit specific conversational behaviors.

\section{$\underline{\text { Research Implications }}$}

Further investigation of pragmatic conversational development is indicated. In addition to this study which assessed normal and developmentally delayed high school students, studies have assessed pragmatic conversational skills of normal, articulation-disordered, and language-disordered second- through fifth-grade students (Hassan, 1982; Lucas, 1983). Similar research with other populations such as children with autism, children with retardation, and children with hearing impairment would add to current knowledge about pragmatic development.

Additional data about the skills of persons with retardation is needed. If this study were to be replicated, the following suggestions may be useful: 1) reorganize the format of the Pragmatic Protocol following the recommendations 
given in chapter IV of this study to allow a more precise quantitative measurement of the subject's behavior, 2) audiotape or videotape the interaction to permit the investigator to replay the interaction when making judgments on appropriateness of behaviors, 3) control variables such as IQ, age, sex, socioeconomic status, and educational background more closely, and 4) compare normally developing children with developmentally delayed children matched for mental age, mean length of utterance, or cognitive level (e.g., using Piaget's, Bruner's, or Guilford's cognitive schemata).

Gathering general data on the development of conversational skills is an important but often elusive task. Since the Pragmatic Protocol gives an overview of a person's conversational skills, it has been a useful research tool. The data gathered in this investigation is preliminary information. Further research is needed in order to gain more knowledge about pragmatic development in normal and language-disordered populations. It is hoped that with this increased knowledge, speech-language pathologists can better intervene to improve the communicative competence of their clients. 


\section{SOURCES CONSULTED}

AUSTIN, J.L. How to Do Things with Words. Cambridge, Mass: Harvard University Press, 1962 .

BATES, E. Language and Context. New York: Academic Press, $1976 \mathrm{a}$.

BATES, E. Pragmatics and sociolinguistics in child language. In D. Morehead and A. Morehead (Eds.), Normal and Deficient Child Language. Baltimore: University Park Press, 1976b.

BATES, E. Intentions, conventions, and symbols. In E. Bates (Ed.), Emergence of Symbols: Cognition and Communication in Infancy. New York: Academic Press, 1979a.

BATES, E. On the evolution and development of symbols. In E. Bates (Ed.), Emergence of Symbols: Cognition and Communication in Infancy. New York: Academic Press, $1979 \mathrm{~b}$.

BATES, P. Effectiveness of interpersonal skills training on the social skill acquisition of moderately and mildly retarded adults. Journal of Applied Behavior Analysis, $1980,13,237-248$.

BEDROSIAN, J., \& PRUTTING, C. Communicative performance of mentally retarded adults in four conversational settings. Journal of Speech and Hearing Research, 1978, 21, 79-95.

BERKSON, G., \& ROMER, D. Social ecology of supervised communal facilities for mentally disabled adults. American Journal of Mental Deficiency, 1980, 85, 219-252.

BEVERIDGE, M. Patterns of interaction in the mentally handicapped. In P. Berry (Ed.), Language and Communication in the Mentally Handicapped. Baltimore: University Park Press, 1976 .

BLOOM, L., \& LAHEY, M. Language Development and Language Disorders. New York: John Wiley and Sons, 1978 .

BRUNER, J. The ontogenesis of speech acts. Journal of Child Language, 1975, 2, 1-20. 
CAMAIONI, L. Child-adult and child-child conversations: An interactional approach. In E. Ochs and B. Schieffelin (Eds.), Developmental Pragmatics. New York: Academic Press, 1979 .

CHAPEY, R. The assessment of language disorders in adults. In R. Chapey (Ed.), Language Intervention Strategies in Adult Aphasia. Baltimore: Williams and Wilkins, 1981.

DALE, P. Language Development: Structure and Function. New York: Holt, Rinehart, and Winston, 1976.

DESILVA, R., \& FAFLAK, P. From institution to community--a new process. Mental Retardation, 1976, 14, 25-28.

DORE, J. A pragmatic description of early language development. Journal of Psycholinguistic Research, 1974, 4, $343-350$.

DORE, J. Holophrases, speech acts, and language universals. Journal of Child Language, 1975, 2, 21-40.

DORE, J. Oh, them sheriff: A pragmatic analysis of children's responses to questions. In S. Ervin-Tripp and C. Mitchell-Kernan (Eds.), Child Discourse. New York; Academic Press, 1977.

DORE, J. Conversational acts and the acquisition of language. In E. Ochs and B. Schieffelin (Eds.), Developmental Pragmatics. New York: Academic Press, 1979 .

EISENSON, J. Stuttering as perseverative behavior. In J. Eisenson (Ed.), Stuttering: A Second Symposium. New York: Harper and Row, 1975.

ERVIN-TRIPP, S. Wait for me, roller skate! In S. Ervin-Tripp and C. Mitchell-Kernan (Eds.), Child Discourse. New York: Academic Press, 1977.

FAINE, S., \& LONGTIN, S. A case study of a Down's Syndrome child's discourse abilities. Queen's College Working Papers in Experimental Speech Pathology and Audiology, 1981, 10, 77-95.

GROSSMAN, H. Manual on Terminology and Classification in Mental Retardation. Baltimore: American Association on Mental Deficiency, 1973.

HALFOND, P., \& TAMARI, P. A method of pragmatic analysis for a Down's Syndrome child. Working Papers in Experimental Speech-Language Pathology. Queen's College of the City University of New York, 1980, 9, 2-19. 
HALLIDAY, M.A.K. Learning How to Mean: Explorations in the Development of Language. New York: Elsevier North Holland, 1977.

HALLIDAY, M.A.K. Language as Social Semiotic: The Social Interpretation of Language and Meaning. Baltimore: University Park Press, 1978.

HARPER, R., WIENS, A., \& MATARAZZO, J. Nonverbal Communication: The state of the Art. New York: John wiley and Sons, 1978 .

HASSAN, P. A Clinical Appraisal of Pragmatic Behaviors. Unpublished master's thesis, University of California, Santa Barabara, 1982 .

HOLLAND, A. Communicative Abilities in Daily Living. Baltimore: University Park Press, 1980.

HOLLIS, J. Communication within dyads of severely retarded children. American Journal of Mental Deficiency, 1966, $70,729-744$.

HYMES, D. Competence and performance in linguistic theory. In R. Huxley and E. Ingram (Eds.), Language Acquisition: Models and Methods. New York: Academic Press, 1971.

JAMES, S., \& SEEBACH, M. The pragmatic function of children's questions. Journal of speech and Hearing Research, $1982,25,2-\overline{11}$.

KRAUSS, R., \& GLUCKSBERG, S. The development of communication: Competence as a function of age. Child Development, $1969,40,255-266$.

LACKNER, J. A developmental study of language behavior in retarded children. In $D$. Morehead and $A$. Morehead (Eds.), Normal and Deficient Child Language. Baltimore: University Park Press, 1976.

LIEBMAN, E. The Descriptive Function of Language: Changes with Age and Pressure to Inform. Unpublished master's thesis, Portland State University, Portland, Oregon, 1982 .

LONGHURST, T. Assessing and increasing descriptive communication skills in retarded children. Mental Retardation, 1972 , $10,42-45$.

LONGHURST, T. Communication in retarded adolescents. Ameri-

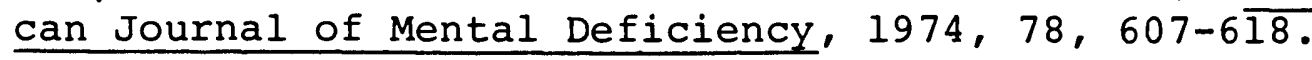


LONGHURST, T., \& BERRY, G. Communication in retarded adolescents: Response to listener feedback. American Journal of Mental Deficiency, 1975, 80, 158- $\overline{164}$.

LUCAS, K. Pragmatic Deficits in Normal, Articulation-Disordered, and Language-Delayed Subjects. Unpublished master's thesis, Portland state University, Portland, Oregon, 1983.

MCDEVITT, S., SMITH, P., SCHMIDT, D., \& ROSEN, M. The deinstitutionalized citizen: Adjustment and quality of life. Mental Retardation, 1978, 16, 22-24.

MCLEAN, J.E., \& SNYDER-MCLEAN, L.K. A Transactional Approach to Early Language Training. Columbus, Ohio: Charles E. Merrill Publishing Co., 1978 .

MILLER, L. Pragmatics and early childhood language development: Communicative interactions in a half-hour sample. Journal of Speech and Hearing Disorders, 1978, 43, 419436 .

MUMA, J.R. Language Handbook. Englewood Cliffs, New Jersey: Prentice-Hall, Inc., 1978 .

MYERS, T. Verbal and nonverbal interactivity. In T. Myers (Ed.), The Development of Conversation and Discourse. Edinburgh: Edinburgh University Press, 1979.

OCHS, E. Introduction: What child language can contribute to pragmatics. In E. Ochs and B. Schieffelin (Eds.), Developmental Pragmatics. New York: Academic Press, 1979.

OWINGS, N., \& MCMANUS, M. An analysis of communication functions in the speech of a deinstitutionalized adult mentally retarded client. Mental Retardation, 1980, 18, 309-314.

PRUTTING, C. Pragmatics as social competence. Workshop presented at Portland State University. Portland, Oregon, March, 1982a.

PRUTTING, C. Pragmatics as social competence. Journal of Speech and Hearing Disorders, 1982b, 47, 123-134.

PRUTTING, C. Pragmatic Protocol and Pool of Pragmatic Behaviors. Santa Barbara, California: Carol Prutting, 1982C.

REES, N. Pragmatics of language: Applications to normal and disordered language development. In R. Schiefelbusch (Ed.), Bases of Language Intervention. Baltimore: University Park Press, 1978. 
REYNOLDS, W.M., \& REYNOLDS, S. Prevalence of speech and hearing impairment of noninstitutionalized mentally retarded adults. American Journal of Mental Deficiency, $1979,84,62-66$.

ROBINSON, H., \& ROBINSON, N. The Mentally Retarded Child. New York: McGraw-Hill, 1965 .

ROM, A., \& BLISS, L. A comparison of verbal communicative skills of language impaired and normal speaking children. Journal of Communication Disorders, 1981, 14, 133-140.

SEARLE, J.R. Speech Acts: An Essay in the Philosophy of Language. Cambridge: Cambridge University Press, 1969.

SEIBERT, J.M., \& OLLER, D.K. Linguistic pragmatics and language intervention strategies. Journal of Autism and Developmental Disorders, 1981, 11, 75-88.

SHATZ, M., \& GELMAN, R. The development of communication skills: Modifications in the speech of young children as a function of listener. Monographs of the Society for Research in Child Development, serial number 152, 1973, 38.

SPRADLIN, J.E., GIRARDEAU, F.L., \& CORTE, H. Social and comunication behavior of retarded adolescents in a twoperson situation. American Journal of Mental Deficiency, $1967,72,473-481$.

TAMARI, P. Language Acquisition of Down's Syndrome Children: The Development of Form and Meaning. Unpublished doctoral dissertation, Teacher's College, Columbia University, 1978 .

WIIG, E. Let's Talk. Columbus, Ohio: Charles E. Merrill, 1983 .

ZIMMERMAN, A. Pragmatics: The Verbal Expression of Feelings. Unpublished master's thesis, Portland State University, Portland, Oregon, 1982. 


\section{APPENDIX A}

October, 1982

Dear student,

I am a graduate student in Speech and Hearing at Portland State University and am doing research for my Master's thesis. The purpose of the study is to describe the language skills involved in a conversation.

In order to complete this project, I need to observe people talking casually. It is hoped that the results of this study may indicate what language skills are needed to hold a conversation. Your participation is voluntary, but I feel that this is a fun subject and you will enjoy it. All that is required is that you talk with a graduate student for 10-15 minutes. In addition, your hearing will be screened.

Your name will not be used in any way and all information will be kept confidential. You are free to withdraw from the study at any time.

If you want to be in the project, sign this form and give it to your teacher. If you have any questions, please feel free to call me at 235-3214 (evenings). Thank you for your interest and time.

Sincerely,

Jane Nicholson

I agree to be in the project and have my hearing screened. 


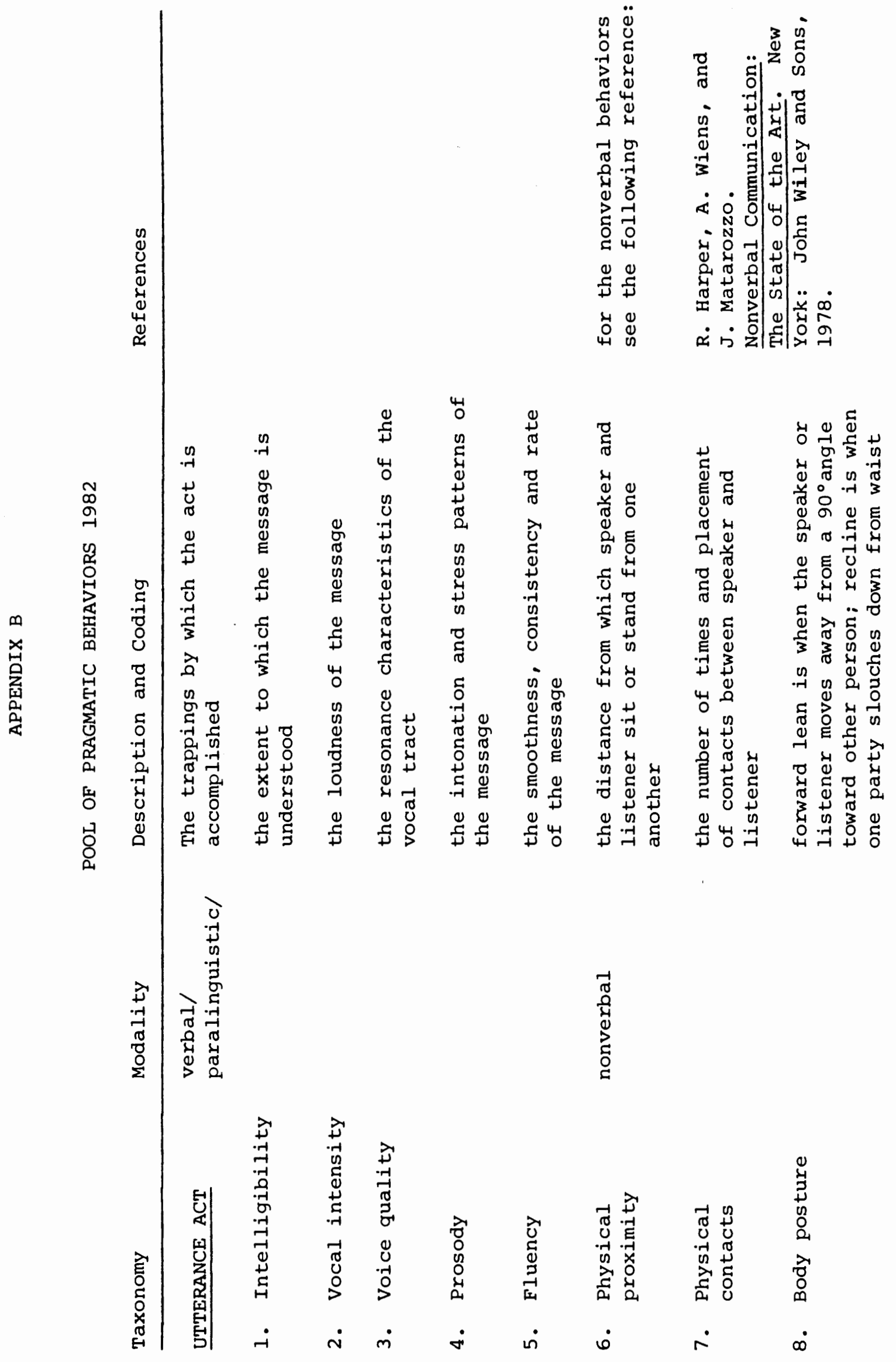




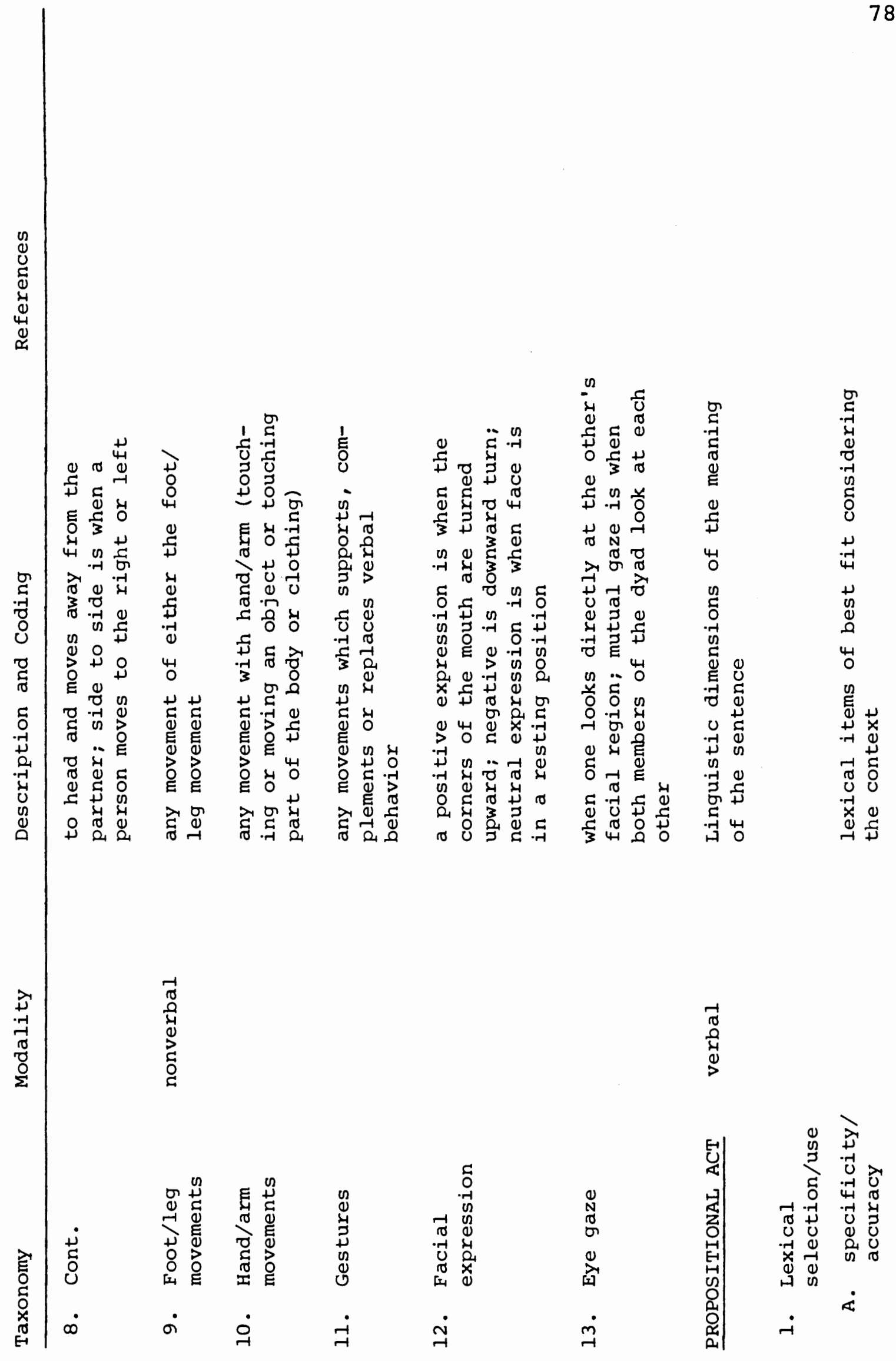




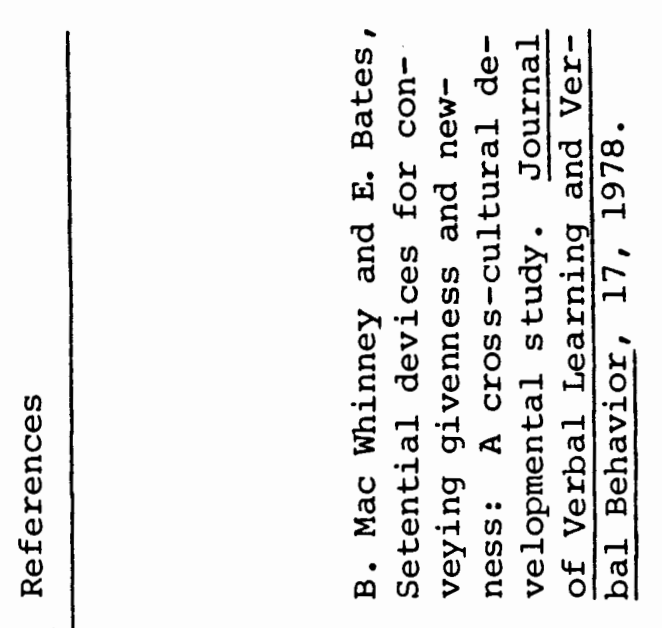

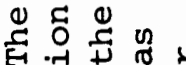

0
0
-1
01
0
0
0
0
4
0
4
4
0
0
4
0
0
0
0
3
7
0
0
-1
+0
0
0
0
0
0
0
0
0
0

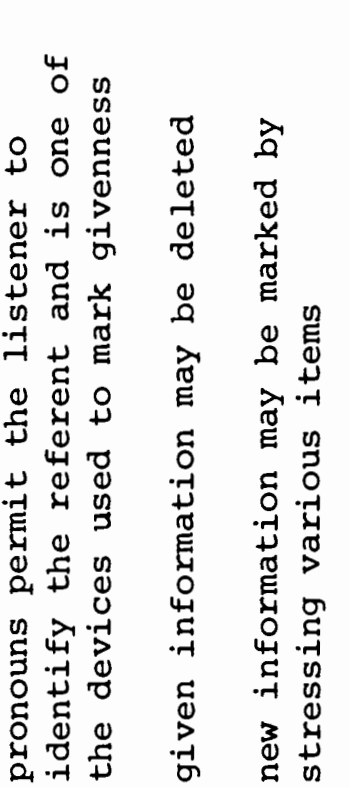

站苗

व 4

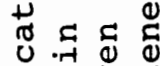

¿ं.न

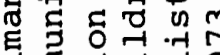

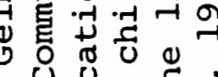

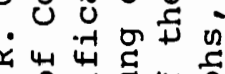

○. Эु

ॠ艺政

N $\tilde{O}_{10}$..

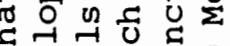

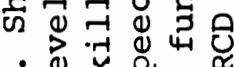

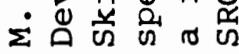

...

है ه

क

थ $\supset$ कृ के

n

ธิ

덩ㄷㅇ

苟苛

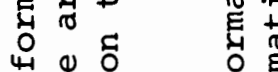

幽

उ.

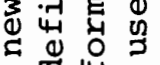

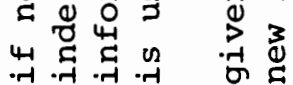
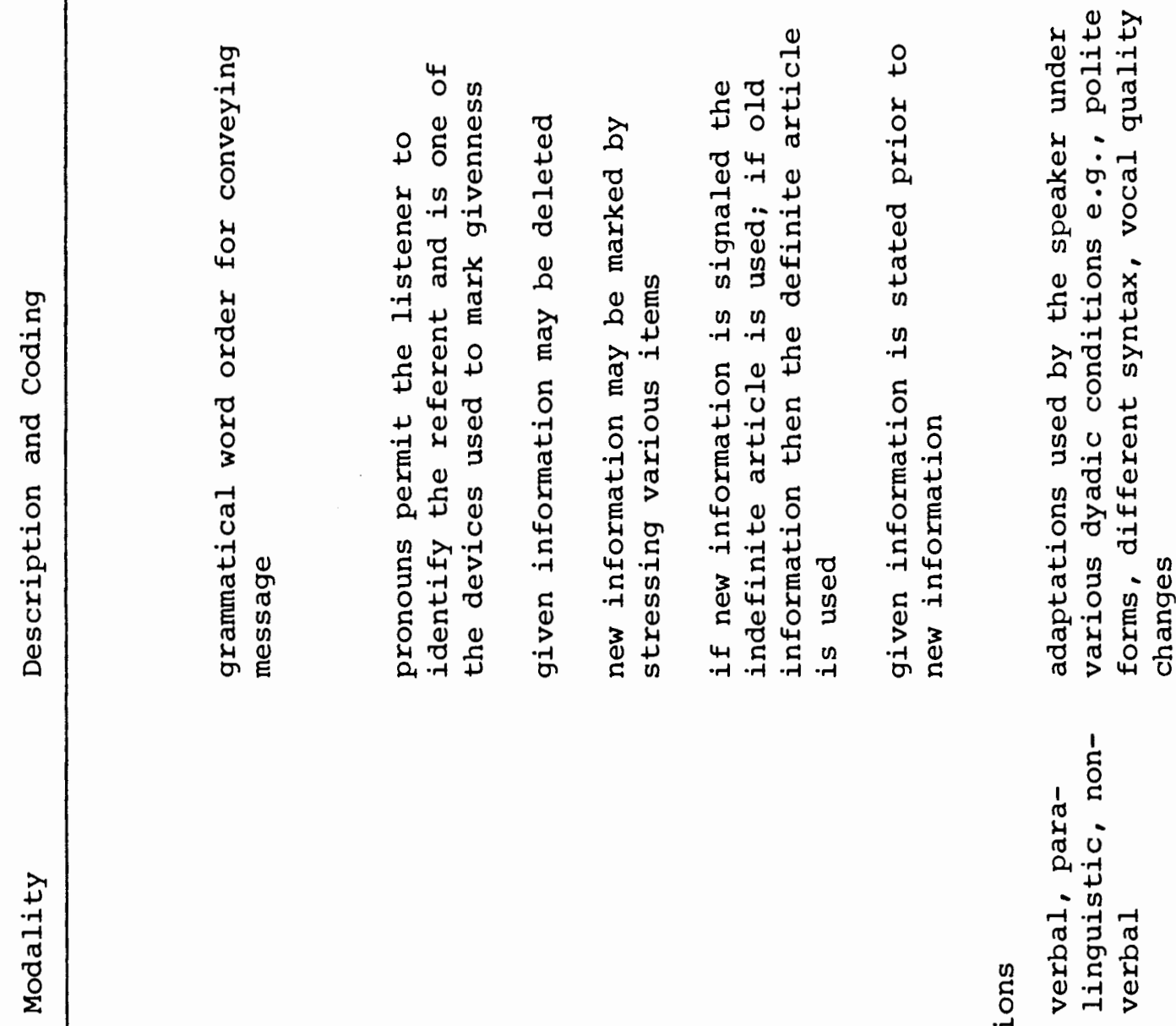

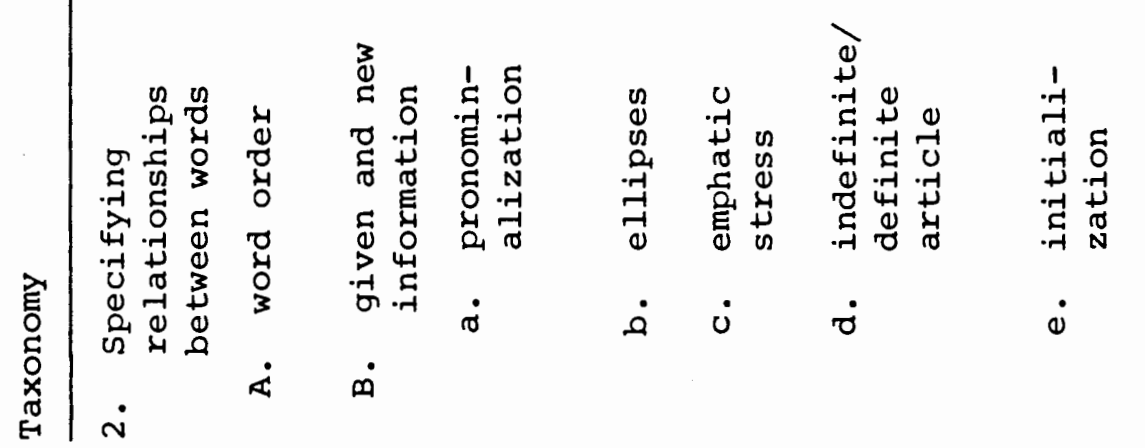

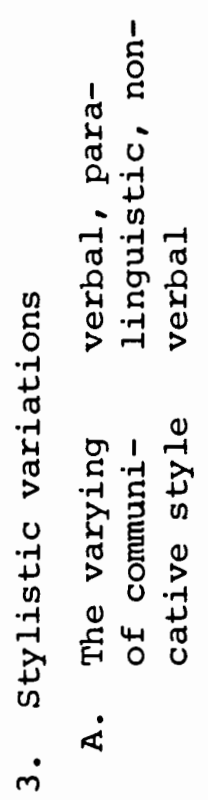



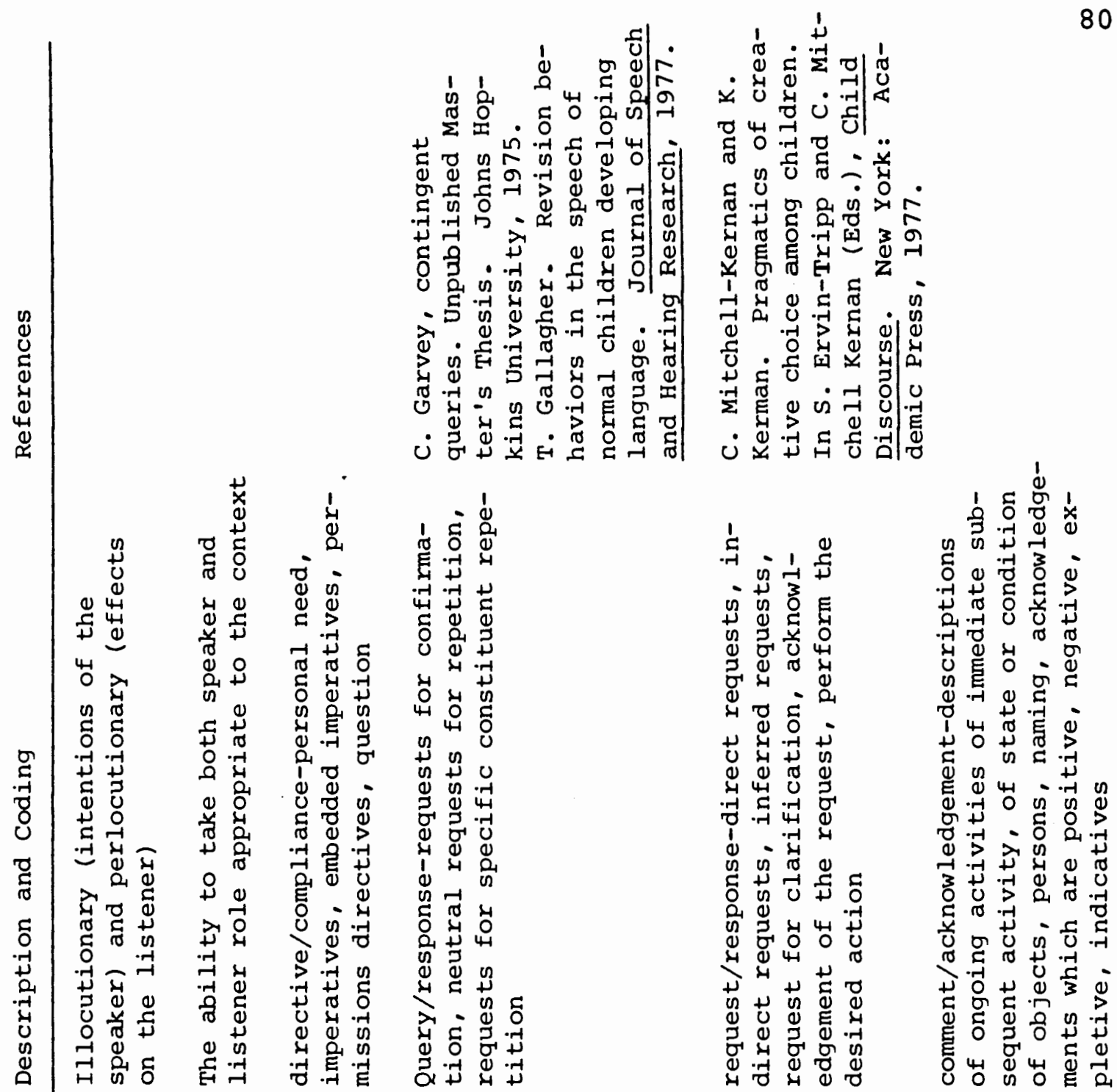


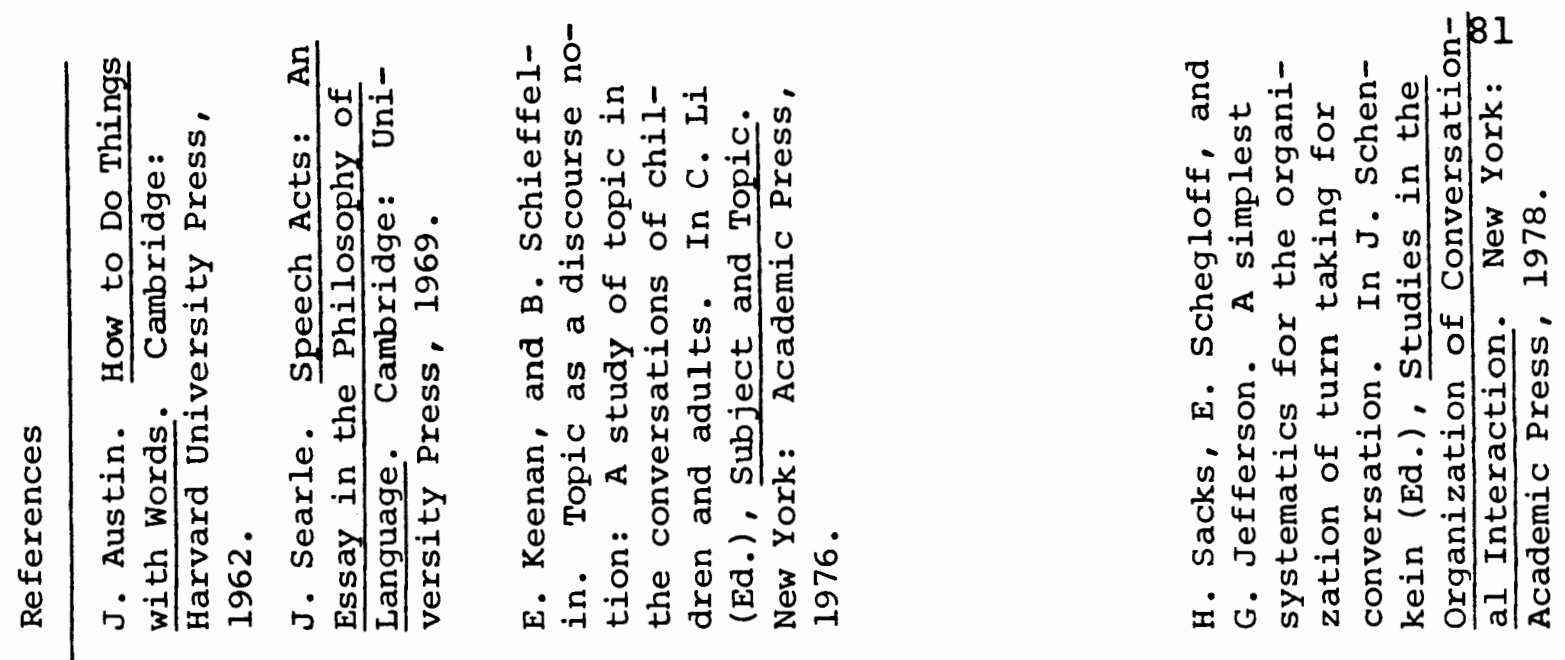

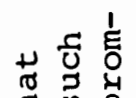

兵 की

प

ป

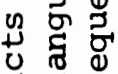

त $\rightarrow$

등.

d

要

प ह त

-

0
+0
0
-1
0
0
0
0
0
2
0
0
0
-1
0
0
+1

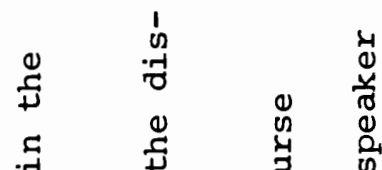

$>+$

헝웜

苟吉

$>00$

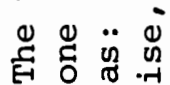

4

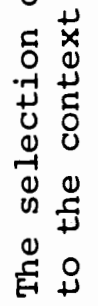

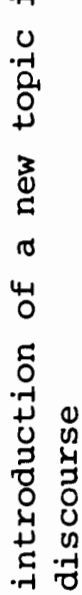

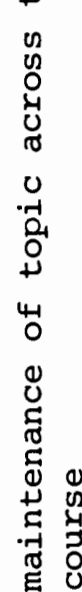

O

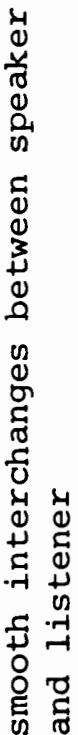

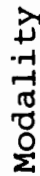

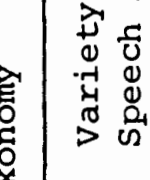

㟧管

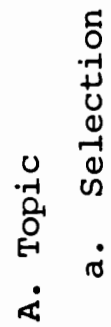

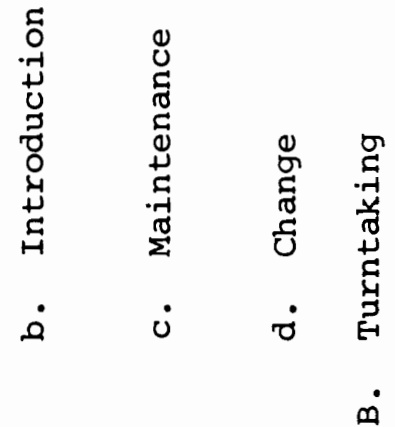




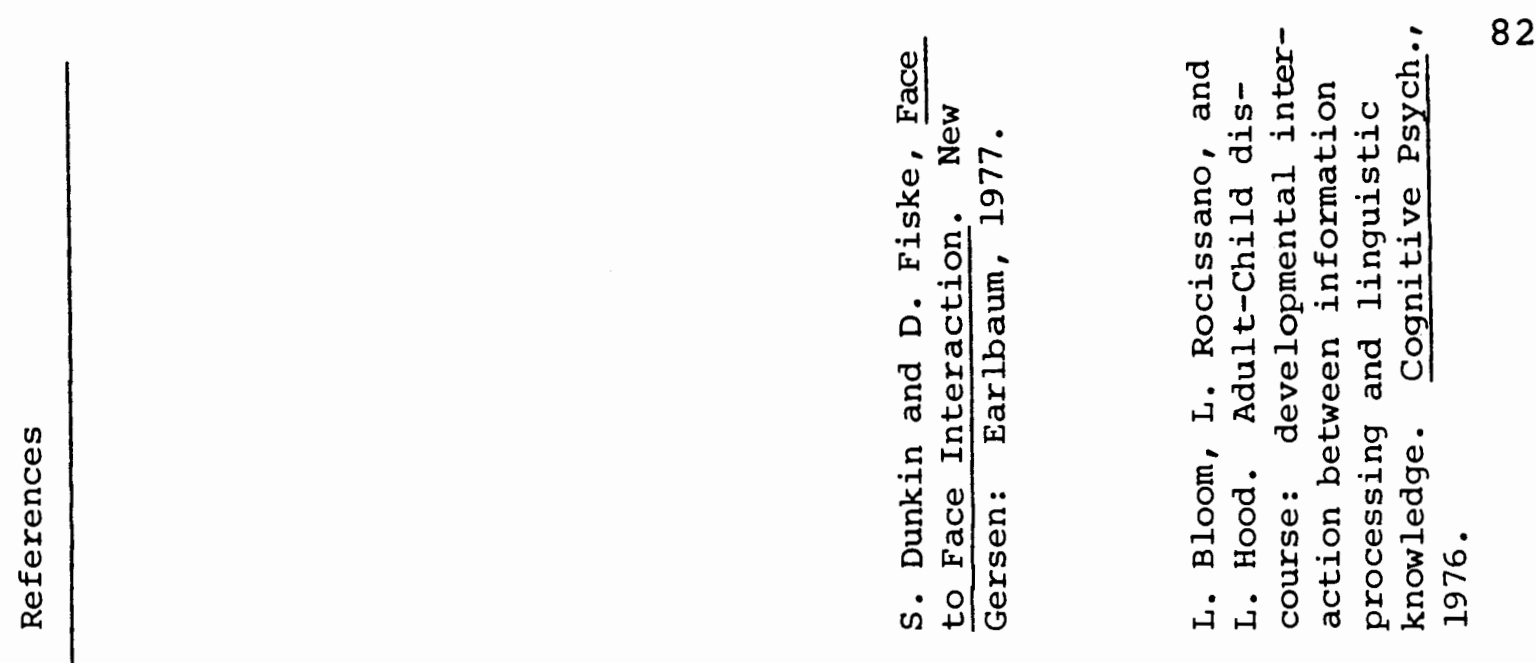




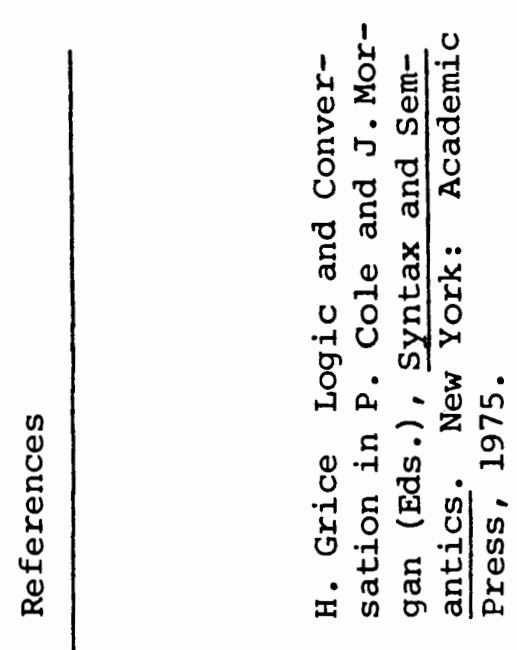

ชُ

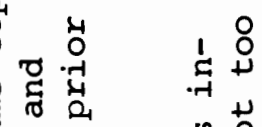

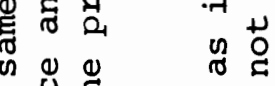

选至

का

+5 \%

$4 \pm c$

药

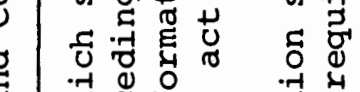

施

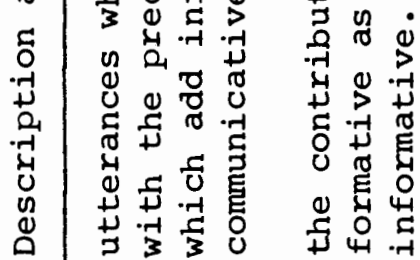

1
-7
7
0
0
0
$\Sigma$

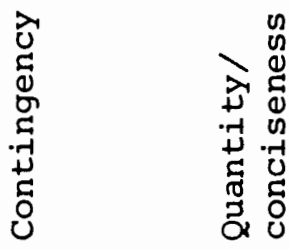

ن

동 :

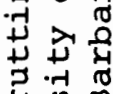

क्ष की

म.

i.

; 点 
PRAGMATIC PROTOCOL

Prutting, 1982

Name :

Date:

Communicative setting

observed:
Communicative Patners

Relationship:

\begin{tabular}{|c|c|c|c|}
\hline Communicative Act & Appropriate & Inappropriate & $\begin{array}{l}\text { No Opportunity } \\
\text { to Observe }\end{array}$ \\
\hline
\end{tabular}

UTTERANCE ACT

A. Verbal/Para-

linguisitc

1. Intelligibility

2. Vocal intensity

3. Voice quality

4. Prosody

5. Fluency

B. Nonverbal

1. Physical proximity

2. Physical contacts

3. Body posture

4. Foot/leg movements

5. Hand/arm movements

6. Gestures

7. Facial expression

8. Eye gaze 
PROPOSITIONAL ACT

A. Lexical selection/ Use

1. Specificity/accuracy

B. Specifying relationships between words

1. Word order

2. Given and new information

C. Stylistic variations

1. The varying of communicative style

ILLOCUTIONARY AND PERLOCUTIONARY ACTS

A. Speech acts

1. Speech act pair analysis

2. Variety of speech acts

B. Topic

1. Selection

2. Introduction

3. Maintenance

4. Change

C. Turntaking

1. Initiation

2. Response

3. Repair/revision

4. Pause time

5. Interruption/ overlapping 


\begin{tabular}{|c|c|c|c|}
\hline Communicative Act & Appropriate & Inappropriate & $\begin{array}{c}\text { No Opportunity } \\
\text { to Observe }\end{array}$ \\
\hline
\end{tabular}

6. Feedback to speaker

7. Adjacency

8. Contingency

9. Quantity/conciseness

C.A. Prutting University of California Santa Barbara 1982 


\section{APPENDIX D \\ Criteria for Evaluating Behaviors on Pragmatic Protocol}

According to evaluation procedures, a subject is placed in a conversational setting with another person for 10-15 minutes. The communicative setting and the relationship between the subject and the interactor are recorded on the protocol form. An evaluator observes the interaction between the subject and interactor and judges the subject's behaviors according to the parameters designated on the Protocol. After the interaction is completed, the evaluator records the subject's behaviors as appropriate, inappropriate, or not observed during the interaction. If the behaviors are marked "not observed," the interaction is continued or repeated until a judgment of appropriate or inappropriate is made on all of the 32 behaviors. In her thesis, Hassan (1982) gives the following guidelines for judging the behaviors qualitatively:

1. Behaviors are marked appropriate if they generally facilitate the interaction at hand and do not appear to be penalizing the child's communicative competence given the overall exchange being observed.

2. Behaviors are judged inappropriate if they appear to be detracting from a smooth communicative exchange and inhibiting the child's perceived communicative competence.

3. Behaviors that are appropriately omitted are judged appropriate; behaviors that are inappropriately omitted are labelled inappropriate. 
4. When the evaluator does not have sufficient information to judge them as either appropriate or inappropriate, behaviors are entered in the no opportunity to observe column. Behaviors entered in this column are reassessed during additional samples of conversation until the evaluator is able to judge them as either appropriate or inappropriate.

It is emphasized that the setting, relationship between partners, and ages of partners be considered when making a judgment as to whether a behavior is appropriate or inappropriate. Examples of appropriate and inappropriate behaviors for each of the 32 parameters are given in Appendix E. 


\section{APPENDIX E}

\section{EXAMPLES OF APPROPRIATE AND INAPPROPRIATE \\ BEHAVIORS FROM THE PRAGMATIC PROTOCOL}

$\overline{\text { Communicative Act }}$ Appropriate

\section{UTTERANCE ACT}

A. Verbal/Para-

linguistic

1. Intelligibility

2. Vocal intensity

3. Vocal quality

4. Prosody

5. Fluency
No difficulty understanding subject's speech. No need to often ask subject to repeat due to poor intelligibility.

Subject uses intensity varied and suited to the message and situation, e.g., quiet when telling secret and louder when excited.

Subject uses vocal quality not distracting to interactor.

Subject uses suitable intonation and stress ing for interactor. patterns not distract-

Subject's speech difficult to understand. Interactor needs to ask subject frequently to repeat due to poor intelligibility.

Subject uses intensity too loud or too soft for interactor and situation.

Subject uses vocal quality distracting to interactor, e.g., harshness, hoarseness, vocal fry, hypernasality.

Subject uses unsuitable intonation and stress patterns distracting for interactor, e.g., monotone, too much variety, not stressing content words.

Subject delivers mesSubject consistently desage smoothly, consistently, and with appropriate rate, e.g., not too fast nor too livers message disfluently, in a "choppy" manner, and/or too fast or too slow for the interactor. slow for the interactor. 
Communicative Act

B. Nonverbal

1. Physical proximity

2. Physical contacts

3. Body posture

4. Foot/leg movements

5. Hand/arm movements

6. Gestures

7. Facial expression

Subject uses variety of facial expressions complementing intent of message and matching situation.
Subject positions self at distance suitable for situation, e.g., in a dyad, within 3-5

feet of interactor.

Subject displays type and amount of physical contact suitable for situation and relationship.

Subject maintains body posture not distracting to interactor.

Subject displays foot/ leg movement appropriate and not distracting to interactor.

Subject displays hand/ arm movement appropriate and not distracting to interactor.

Subject uses suitable gestures for accompanying verbal message.

Inappropriate

Subject displays type and amount of physical contact unsuitable for situation and relationship, e.g., patting interactor on head, kissing unfamiliar interactor.

Subject uses body posture distracting to interactor, e.g., "slouching," rocking.

Subject displays foot/ leg movement distracting to interactor, e.g., putting feet on table or constantly kicking table with feet.

Subject displays hand/ arm movement distracting to interactor, e.g., putting hands in mouth, picking nose, playing with hair with hands.

Subject uses gestures distracting to interactor, e.g., using gestures in place of speaking, using gestures conflicting with meaning of verbal message.

Subject uses facial expressions distracting to interactor or not fitting message, e.g., no change in expression, smiling while telling sad story. 


\section{Communicative Act}

8. Eye gaze
Appropriate

Subject looks at inter- Subject does not look at actor's facial region interactor's facial refor appropriate amounts gion for appropriate of time during interchange.

Subject uses vocabulary fitting the situation and accurate enough to convey message.

Subject consistently uses accurate word order.

Subject signals and marks given and new information accurately for interactor, e.g. uses pronouns, ellipses, emphatic stress.
Subject uses vocabulary interfering with communication, e.g., vague terms such as "thing," swear words offending to interactor.
2. Given and new information
B. Specifiying relationships between words

1. Word order

\section{Stylistic Variations}

I. The varying of communicative style
Subject uses style suitable for relationship with interactor.
Subject consistently uses inaccurate word order which detracts from intelligibility of message for interactor, e.g., "I store went."

Subject does not signal and mark given and new information accurately for interactor, e.g., using pronoun "he" before telling who "he" is; using a person's name when a pronoun could be used.

Subject does not use style suitable for relationship with interactor. 


\section{Communicative Act \\ ILLOCUTIONARY / PERLO- \\ CUTIONARY ACT}

A. Speech act

1. Speech act pair analysis

\section{Variety of speech acts}

\section{B. Topic}

\section{Selection}

2. Introduction

3. Maintenance

4. Change
Appropriate

Inappropriate subject takes both the speaker and listener role in the interchange.

Subject uses language for a variety of functions which suit the situation.

Subject selects topic suitable and relevant for interchange.

Subject introduces topic in a logical, coherent way.

Subject maintains his topic and interactor's topic during interchange.

Subject marks change of topic effectively for interactor, alerting him that he has changed topic.
Subject does not take both speaker and listener roles, e.g., dominates conversation by always taking speaker role; listens and only responds to direct questions.

Subject does not use adequate variety of functions, e.g., predominately uses one function such as requesting.

Subject does not select topic suitable for interchange, e.g., talks about something boring to interactor.

Subject does not introduce topic effectively, e.g., jumps abruptly from topic to topic.

Subject does not maintain his nor interactor's topic during interchange, e.g., answers interactor's questions, but does not expand on them to further discussion of topic.

Subject changes topic, but does not alert interactor that he has changed topic. 


\section{Communicative Act \\ C. Turntaking}

1. Initiation

2. Response

3. Repair/revision

4. Pause time

5. Interruption/ overlap

6. Feedback to speaker

7. Adjacency
Subject initiates

topics and statements.
Subject does not initiate topic and statements without prompts from interactor, e.g., only responds to interactor's questions and statements.

Subject does not reply to interactor's statements and questions. or not on topic).

When interactor does not understand message, subject clarifies or repairs own statement. When subject does not understand interactor's message, subject asks interactor to clarify.

Subject uses adequate amount of pause time between words and times response to interactor appropriately.

Subject rarely interrupts interactor.

Subject gives interactor verbal and nonverbal feedback that he is listening, e.g., "yeah," "really," nodding.

Subject gives utterances which fit immediately prior utterance.
During communication breakdown, subject does not repair own statements nor request clarification of interactor's statements.

Subject does not use adequate amount of pause time between words and times responses to interactor inappropriately, e.g., too long or too short.

Subject frequently interrupts interactor.

Subject does not give interactor any feedback that he is listening to what interactor is saying.

Subject gives utterances which do not fit immediately prior utterance, e.g., answering questions. much later with intervening conversation between question and response. 


\begin{tabular}{|c|c|c|}
\hline Communicative Act & Appropriate & Inappropriate \\
\hline 8. Contingency & $\begin{array}{l}\text { Subject responds to } \\
\text { immediately prior } \\
\text { utterance and expands } \\
\text { on it, e.g., "Let's go } \\
\text { to the store." "Yeah, } \\
\text { and let's get apples." }\end{array}$ & $\begin{array}{l}\text { Subject does not expand } \\
\text { on prior utterance, e.g., } \\
\text { repeats interactor's } \\
\text { statements but never } \\
\text { adds information: "You } \\
\text { went to the beach?" } \\
\text { "Yeah, I went to the } \\
\text { beach." }\end{array}$ \\
\hline $\begin{array}{l}\text { 9. Quantity/ } \\
\text { conciseness }\end{array}$ & $\begin{array}{l}\text { Subject consistently } \\
\text { gives enough but not } \\
\text { too much information } \\
\text { to the interactor. }\end{array}$ & $\begin{array}{l}\text { Subject consistently } \\
\text { contributes too much or } \\
\text { not enough information } \\
\text { for the interactor. }\end{array}$ \\
\hline
\end{tabular}

K. Lucas and J. Nicholson

Portland State University

Portland, Oregon 1983 


\section{APPENDIX F}

\section{SEATING ARRANGEMENTS}

General Floor Plans:

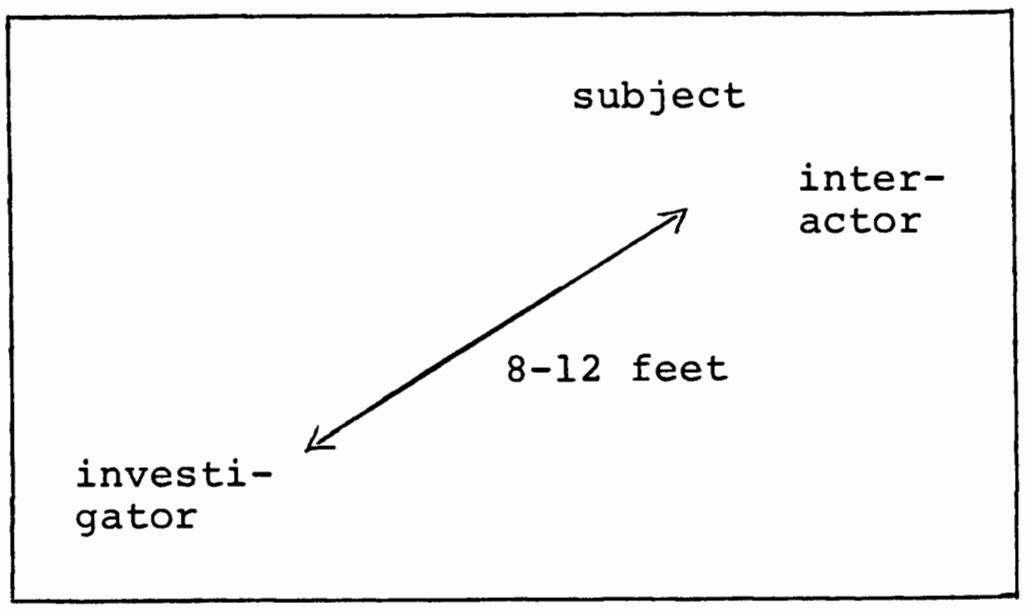




\section{APPENDIX G}

\section{SCRIPT}

Strategies used by the interactor to potentially elicit some specific pragmatic behaviors from the subject.

$\underline{\text { Strategy }}$

(Observed when first introduced and when leaving)

Early in the conversation, the interactor will look at a piece of the Subject's clothing and say "Gee, I like your "Where did you get it?"

During a pause in the discourse the interactor wili remain quiet but maintain eye gaze toward the subject. If, after 15 seconds, the subject has not introduced a topic, the interactor will begin the conversation.

After the subject makes a statement, on one occasion the interactor will repeat the statement incorrectly and with a rising inflection at the end of the phrase. If the subject doesn't correct the interactor, the interactor will ask "What is that?/What do you mean?/I don't understand." at another time during the discourse.
Pragmatic Behavior(s)

Variety of Speech Acts (\#19) Greetings and Salutations

Speech act pair analysis (\#18) -- acknowledgement of a comment

Turn taking (\#25) -- response Speech act pair analysis (\#18) -- response to a query

Turntaking (\#24) -- initiation

Topic -- (\#20) selection (\#21) introduction (\#23) change

Turntaking (\#26) -- repair/ revision

Speech act pair analysis (\#18)

- response to request for clarification

Variety of speech acts (\#19) -- protest
At one point during the conversation, the interactor will
Speech act pair analysis (\#18) -- request for clarification 
lower her voice and "mumble."

If the subject doesn't ask

for clarification, the interactor will repeat the strategy at one other time during the discussion. 


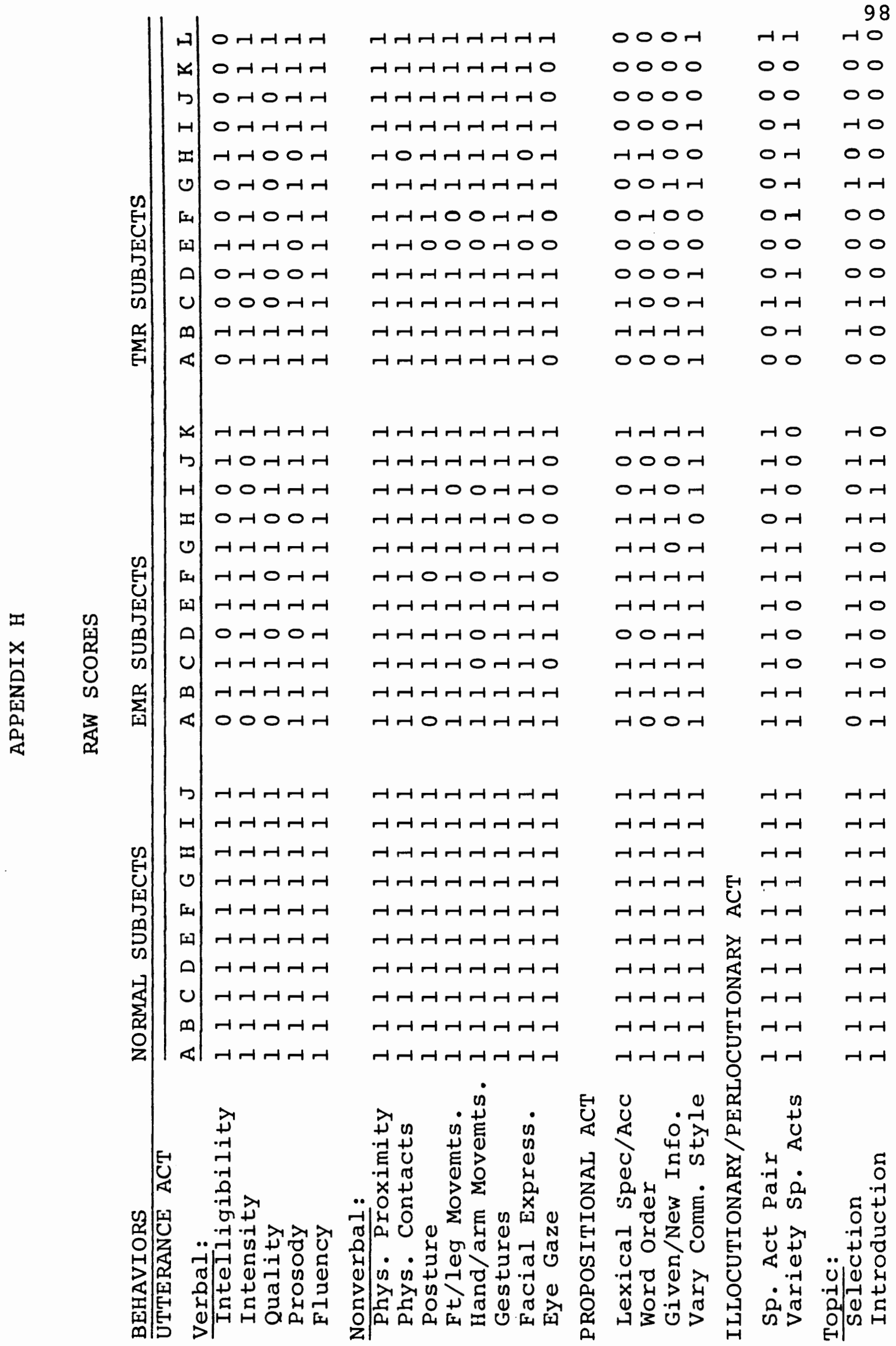




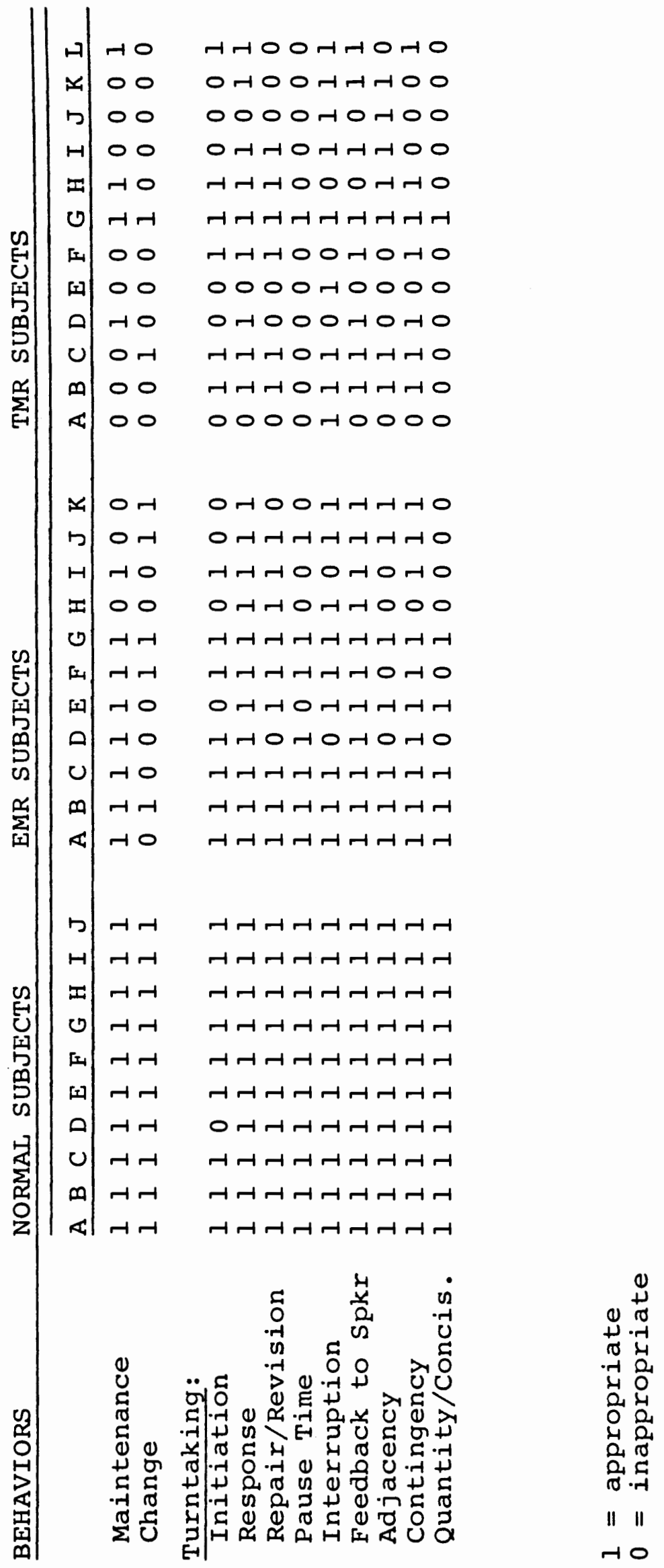

